\title{
Effect of Statistical Counting Errors on Wavelength Criteria for X-Ray Spectra
}

\author{
John S. Thomsen' and F. Y. Yap ${ }^{2}$
}

(June 29, 1967)

\begin{abstract}
Various features of the spectral profile of an x-ray line can be measured with an uncertainty which is only a small fraction of the observed line width. With recent improvements in measurement techniques, statistical errors due to the random fluctuations of the intensities in counter recordings may become significant. The present study considers the effect of such errors on several features of the line profile which could be used for definition of its wavelength. These may be broadly classified into three groups, viz, the peak, the centroid, and the median. In the present analysis the statistical errors associated with these features are compared theoretically, with the assumption of negligible error in angular measurement. Certain systematic errors are also briefly examined. The effects of truncation range, asymmetry, and background intensity are considered, as well as possible optimization of the data-taking procedure.

In general, $\sigma$, the standard deviation of the wavelength, is given by $\sigma / W=F /\left(I_{p} T\right)^{1 / 2}$, where $W$ is the full width at half-maximum intensity, $I_{p}$ the peak intensity, $T$ the total counting time, and $F$ a dimensionless factor of the order of unity. Thus $F$ may be regarded as a factor of merit for comparing the various cases, a low value of $F$ being desirable. When the form of the line profile is known a priori, it is usually best to make use of this knowledge; e.g., a Lorentzian can be thus fitted with $F \approx 0.8$ for any of the three wavelength features. Using optimized truncation ranges and including the error in locating end points, one obtains approximately this same $F$ for the centroid or median even without prior knowledge of the profile. In the latter case the value of $F$ for the peak usually ranges from about 1.6 to 2.1. However, the peak is less subject to certain systematic errors and is preferable from the viewpoint of simplicity and historical precedent. It is recommended that use of the peak be continued at present; further study of the problem from the viewpoint of atomic energy level interpretation would be desirable.
\end{abstract}

Key Words: Lattice constants, peak intensity, physical constants, spectral line profiles, statistical error analysis, $x$-ray line shapes, x-ray spectra, x-ray wavelength criteria.

1. Introduction.

1.1. Problem of wavelength criterion

1.2. Previous investigations.

188

1.3. Profile features for wavelength criteria............... 189

2. Preliminary considerations................................ 189

2.1. Experimental assumptions............................. 189

2.2. Lorentzian line profile............................... 190

2.3. Asymmetric lines..................................... 190

2.4. Gaussian profile.......................................... 191

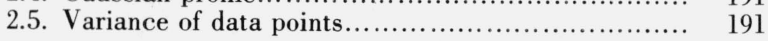

3. Centroid................................................. 191

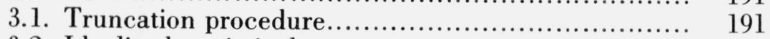

3.2. Idealized statistical error................................ 192

3.3. Optimization of statistical error........................... 192

3.4. Truncation mislocation factor............................ 192

3.5. Application to quasi-Lorentzian profile................ 193

3.6. Application to quasi-Gaussian profile.................... 194

3.7. Systematic errors ..................................... 194

3.8. Effect of asymmetry ......................................

4. Median.

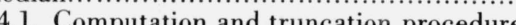

*An invited paper. This work supported in part by the National Bureau of Standards. ${ }^{1}$ Department of Physics, Johns Hopkins University, Baltimore, Md. 21218.

${ }^{2}$ Physics Department, Wilson College, Chambersburg, Pa. 17201.

\section{Contents}

\section{age} 88

88

8

(n)

9

5

(1)

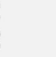

4
\section{} 195

\begin{tabular}{|c|c|}
\hline \multirow{2}{*}{\multicolumn{2}{|c|}{$\mathrm{P}$}} \\
\hline & \\
\hline 4.3. Optimization of statistical error. & 195 \\
\hline 4.4. Truncation mislocation factor.... & 195 \\
\hline 4.5. Application to quasi-Lorentzian profile.. & 196 \\
\hline 4.6. Application to quasi-Guassian profile... & 196 \\
\hline 4.7. Systematic errors..................... & 196 \\
\hline 4.8. Effect of asymmetry................. & 197 \\
\hline Geometrical peak. & 197 \\
\hline 5.1 Orthogonal polynomial technique. & 197 \\
\hline al error........................ & 197 \\
\hline 5.3. Goodness of fit... & 198 \\
\hline tion to quasi-Lorentzian profile......... & 198 \\
\hline 5.5. Optimization of statistical error....................... & 199 \\
\hline 5.6. Application to quasi-Gaussian profiles............... & 199 \\
\hline 5.7. Systematic errors...... & 199 \\
\hline of asymmetry... & 200 \\
\hline 5.9. Parabolic fit.. & 200 \\
\hline 5.10. Optimization of parabolic fit......... & 200 \\
\hline 5.11. Profile known a priori.......... & 201 \\
\hline 5.12. Optimization of Lorentzian.......................... & 201 \\
\hline Extrapolated peak. & 202 \\
\hline 6.1. Midpoint of single $c$ & 202 \\
\hline 6.2. Use of fitted curve. & 202 \\
\hline 6.3. Use of raw data... & 203 \\
\hline lusions. & 203 \\
\hline es & 20 \\
\hline
\end{tabular}




\section{Introduction}

\subsection{Problem of Wavelength Criterion}

X-ray emission lines have natural widths of the order of 0.1 percent of their wavelengths and in many cases show considerable asymmetry in their line profiles [1]. ${ }^{3}$ Precision wavelength measurements [2,3] have frequently claimed accuracies of a few parts per million; in a recent paper by Bearden and coworkers [4] probable errors of approximately one part per million were reported for five standard reference lines. Since the error estimate is thus only a fraction of 1 percent of the line width, it is clearly necessary to use some specific feature of the line profile in defining the wavelength. For symmetric lines all criteria which are likely to be regarded as reasonable definitions will reduce to the same value; in the more general case of asymmetric lines, the resulting values may differ appreciably.

These wavelengths are vitally important both in determining atomic energy levels and in providing standards for crystallography and $\gamma$-ray spectroscopy. Hence at least four factors should be considered in formulating a wavelength criterion, viz:

1. Consistency of the resulting energy level scheme with the Ritz combination principle, i.e., the atomic interpretation.

2. Simplicity of the definition.

3. Historical precedents and current usage.

4. Attainable accuracy as limited by the effects of both systematic and statistical errors.

While the first factor is clearly the one of most fundamental physical significance, presently available experimental data and theoretical knowledge of x-ray line shapes do not appear to provide a clear answer to this point. From the standpoint of simplicity and intuitive appeal the peak of the line profile is probably the most acceptable criterion. This is also generally supported by current usage in x-ray spectroscopy, as well as considerable historical precedent.

The majority of tabulated $x$-ray emission wavelengths were recorded with photographic plates. Usually it was not stated just what point on the profile defined wavelength, although this probably corresponded, at least approximately, to the position of maximum blackening. In some cases a microdensitometer trace of the plates was made and the maximum used to specify wavelength.

Most recent high precision determinations measure the peak of a line by rotating a crystal through small angular increments and recording the intensity at each step by means of a photon counter (gas or scintillation counter), yielding a step-by-step scan of the line profile. This basic method has been used with single-crystal [4], double-crystal [4], and curved-crystal [5] spectrometers. High angular accuracy has long been attainable; the recent development of an angular interferometer [6] has furnished a new precise and convenient technique to facilitate the required angular measurements.

\footnotetext{
${ }^{3}$ Figures in brackets indicate the literature references at the end of this paper.
}

The accuracy of a wavelength measurement is limited by both systematic and statistical errors. Systematic errors may arise from numerous causes, e.g., drift in $x$-ray tube voltage, presence of satellites, and distortion due to the instrumental "window." While this window may be the predominant factor in crystallography and $\gamma$-ray spectroscopy, it is much less serious in $\mathrm{x}$-ray spectroscopic work.

With good crystals and proper geometry, such distortion of the true line profile may be held to a minimum. For example, using two good quartz crystals with the Mo $\mathrm{K} \alpha_{1}$ line in second order, Bearden [4] obtained a window curve which was only about 3 percent of the natural line width. Brogren [7] reported a comparably narrow window with the $\mathrm{Cu} \mathrm{K} \alpha_{1}$ line. Moreover, Bonse and Hart [8] recently reported almost tailless x-ray rocking curves obtained by multiple Bragg reflections between the walls of grooves cut into large perfect crystals of silicon and germanium. Furthermore, one may remove much of the residual instrumental distortion by "unfolding techniques" [9]; Sauder [10] has given a somewhat simplified method of correcting selected features of the line profile.

The statistical error is largely due to random intensity fluctuations, caused by the inherent nature of the photon emission from the x-ray target and by the properties of the counter. The present paper is principally concerned with this factor as it affects the various features used to define wavelength. It also treats briefly certain sources of systematic error which are sensitive to the choice of wavelength criterion.

Thomsen [11] has given a preliminary analysis of the problem and indicated how the errors may often be reduced by varying the counting times for different points on the line profile. Subsequently Wilson, Thomsen, and Yap [12] generalized this optimization procedure. The overall problem has been comprehensively treated in a thesis by Yap [13], which forms the basis for the present paper.

This treatment will consider at length three features of the line profile, viz, the centroid, the median, and the peak. To date the peak has been used almost exclusively in $\mathrm{x}$-ray spectroscopy whenever a defining feature was explicitly specified. Some crystallographers [14] and $\gamma$-ray spectroscopists [15] have employed the centroid and the median in a limited number of cases.

The greater mathematical simplicity of the centroid and median facilitates a more straightforward and complete analysis; hence we will treat them first. Both offer the possibility of a limited improvement in the statistical error. However, we finally recommend continued use of the peak, primarily because of its simplicity and its current acceptance. The statistical error of the peak is treated at length in section 5 , which is independent of the analysis of the centroid and median in sections 3 and 4 .

\subsection{Previous Investigations}

Other investigators have studied various aspects of the problem. Pike and Wilson [16] derived the statis- 
tical error of the centroid; Parrish [14] and his coworkers studied, both theoretically and experimentally, the use of the centroid for lattice parameter measurements, including the effect of truncation error. Merrill and DuMond [17] analyzed the problem of fitting a Lorentzian to the data points by least squares and outlined a method for obtaining the resulting statistical error of the peak. Previously Muller, Hoyt, Klein, and DuMond [18] had derived an expression for the statistical error that arises in fitting any assumed profile to the data and applied this result to the case of an isosceles triangle. Knowles [19] obtained an approximate expression for the statistical error of the median.

Several workers have made comparative studies of various features. Cohen and DuMond [20] discussed the relative merits of the centroid and the peak, and recommended the use of the latter. The same conclusion was reached earlier by Porteus and Parratt [21] in an unpublished investigation. Wilson [22] analyzed both the statistical and the systematic errors that result from locating the peak by a least-squares parabolic fit to the data and by finding the midpoint of a horizontal chord. Beu and Scott [23] discussed reasons for preferring the peak to the centroid for lattice parameter measurements. Recently Bačkovsky [24] compared several features, including the peak and the midpoint of a horizontal chord, and suggested using the latter, with the chord drawn near the inflection point of the line profile.

\subsection{Profile Features for Wavelength Criteria}

a. The Centroid. In the present analysis we will define the centroid $x_{c}$ as the center of mass of some specified truncated portion of the line profile, i.e.,

$$
x_{c}=\int_{X_{1}}^{X_{2}} x I(x) d x / \int_{X_{1}}^{X_{2}} I(x) d x,
$$

where $I(x)$ represents the true intensity and $X_{1}$ and $X_{2}$ the selected truncation limits. In principle, the abscissa variable $x$ should represent wavelength; however, x-ray lines are sufficiently narrow so that an angle or an energy scale may be employed with negligible error.

It should be noted that crystallographers [14] who have employed the centroid have always assumed an infinite range in the above definition (which is essential in order to apply the convolution theorem for addition of centroids). Experimentally it was, of course, necessary to infer the centroid value by extrapolation from a finite truncated portion of the curve. By contrast the present paper considers the possibility of defining the centroid with finite truncation limits, which represents a generalized concept.

b. The Median. Similarly we will define the median as that value $x_{m}$ which equally divides some specified portion of the line profile, i.e.,

$$
\int_{X_{1}}^{x_{m}} I(x) d x=\int_{x_{m}}^{X_{2}} I(x) d x
$$

c. The Peak. Finally we will define the peak simply as the abscissa $x_{p}$ corresponding to the maximum intensity. (In this connection we will disregard the possibility of multiple peaks.) In practice two primary techniques have been used to locate this feature; the results so obtained will be termed (i) the geometrical peak and (ii) the extrapolated peak.

(i) The Geometrical Peak. A smooth curve may be fitted to the raw data points by a computer (using either a theoretical line profile or an empirical function with undetermined parameters) or drawn in graphically. The geometrical peak $x_{p}$ is then defined as the abscissa value for which the maximum occurs, i.e.,

$$
[d I(x) / d x]_{x=x_{p}}=0 .
$$

(ii) The Extrapolated Peak. When graphical methods are used, statistical fluctuations make it difficult to determine the exact maximum of a smooth curve. Hence spectroscopists developed the technique of drawing several horizontal chords across the upper portion of the profile, determining their midpoints, and drawing a smooth "bisector curve" (often simply a straight line) through these midpoints; the bisector was then extrapolated to intersect the line profile to obtain the peak [25]. This method is illustrated in figure 1 . With the advent of computers various analytical modifications of this technique are now feasible; these are described more fully in section 6 . We will denote the peak found by any of these methods as the extrapolated peak.

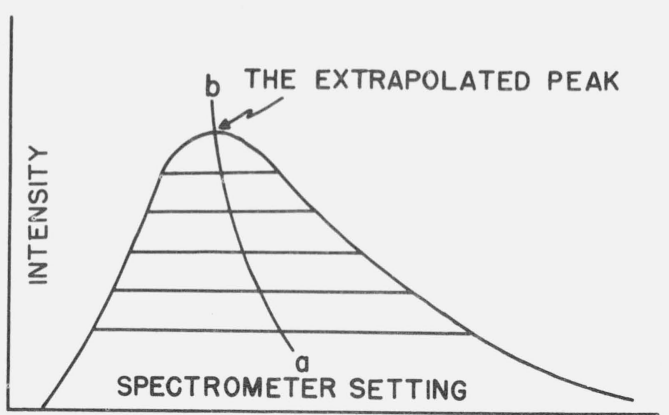

FIGURE 1. Extrapolation technique of locating peak by means of a smooth bisecior curve through chord midpoints.

For symmetric lines it is also possible to use the average of the midpoints of several chords [26] or even the midpoint of a single chord [27] as a means of determining the peak. (Since the lines are assumed symmetric, this technique might equally well be said to determine the centroid or the median.) For reasons of mathematical similarity, this approach is considered along with the extrapolated peak.

\section{Preliminary Considerations}

\subsection{Experimental Assumptions}

Our present analysis is primarily from the viewpoint of the spectroscopist rather than the crystallographer. 
The spectroscopist is chiefly interested in a simple, accurate, reproducible criterion for measuring wavelengths; he normally employs a device with a relatively narrow instrumental window (e.g., a double-crystal spectrometer), which does not greatly distort the natural line profile. The crystallographer uses $\mathrm{x}$ rays as a tool to study crystal structure and frequently employs a powder diffractometer, which greatly broadens the natural line.

These differences may lead to the choice of different wavelength criteria. For example, the crystallographer may employ the centroid and the spectroscopist use the peak. No fundamental difficulty should result, provided that these two features can be related with high accuracy for the rather limited number of intense lines commonly used by crystallographers.

We will adopt the following assumptions as to experimental conditions:

(A) There is no drift in x-ray intensity incident on the spectrometer.

(B) Both counting time intervals and angular positions (and hence the corresponding wavelength or energy scales) on the spectrometer can be measured with negligible error.

(C) The instrumental window causes a negligible shift in the selected feature for the wavelength criterion, or the necessary correction may be made with negligible error.

(D) Measurements are recorded at a finite number of closely spaced points (usually at equal abscissa intervals). Consequently it will always be assumed possible to replace an integral by the corresponding sum, and vice versa, whenever convenient.

(E) Photon counts at the individual data points are statistically independent of one another and follow the Poisson distribution, and the number of counts recorded is always sufficiently large so that the latter may be approximated by a normal distribution. It follows that the variance (i.e., the square of the standard deviation) for a total count $N$ at the $i$ th point is given by

$$
\sigma_{N}^{2}=N
$$

Experimental tests [13] are in good accord with these assumptions.

(F) Background is relatively small compared to the peak intensity and is free from drift. In most of the analysis we will also assume that the background is independent of wavelength over the relevant portion of the profile; however, the effect of a linear variation will also be briefly discussed.

Background intensity $B$ may be determined experimentally by preliminary measurements at points well removed from the peak on either side. This is then subtracted from the experimentally observed value, $I_{\text {obs }}$, for each point to give the computed intensity, i.e.,

$$
I=I_{\mathrm{obs}}-B .
$$

An error in the background correction $B$ may produce a systematic effect throughout a given run, but will not affect the statistical error discussed under assumption $(\mathrm{E})$ above. Hence the effective variance of $I$ is

$$
\sigma^{2}(I)=\sigma^{2}\left(I_{\mathrm{obs}}\right) .
$$

Thus it is governed by the total intensity, both line and background.

\subsection{Lorentzian Line Profile}

There is both theoretical [28] and experimental [29] evidence to indicate that many $x$-ray emission line profiles are closely approximated by a Lorentzian curve (often referred to as a witch), which is given by

$$
I(x)=I_{p}\left[1+\frac{4\left(x-x_{p}\right)^{2}}{W^{2}}\right]^{-1},
$$

where $I$ and $x$ represent intensity and wavelength respectively, $I_{p}$ and $x_{p}$ denote the respective coordinates of the peak, and $W$ represents the full width of the curve at half maximum intensity.

Frequently the Lorentzian curve may be a fair approximation to the true line profile, but not sufficiently good to be assumed a priori. In such cases, after the standard deviation has been calculated in generalized form, we may then insert the Lorentzian expression, eq (2.4), only for the purpose of obtaining an error estimate. We will term such a line profile a quasi-Lorentzian.

It is convenient to normalize $I$ and $x$ in the above expression by defining

$$
\begin{aligned}
& g=I / I_{p}, \\
& v=2\left(x-x_{p}\right) / W .
\end{aligned}
$$

Except where otherwise indicated, all equations will be expressed in terms of these normalized coordinates.

The Lorentzian equation then becomes

$$
g(v)=\left(1+v^{2}\right)^{-1} .
$$

\subsection{Asymmetric Lines}

Many prominent x-ray emission lines are asymmetric; this property has usually been described by an index of asymmetry given by $\left(v_{+}-v_{p}\right) /\left(v_{p}-v_{-}\right)$, where $v_{p}$ is the wavelength of the peak while $v_{+}$and $v_{-}$represent the wavelengths of the half intensity points (i.e., $g_{ \pm}=g_{p} / 2$ ) on the long and short wavelength sides respectively. Under this definition asymmetries ranged from zero to infinity with a symmetric line having a value of unity.

More recently Sauder [10] proposed the definition

$$
s=\frac{\left(v_{+}-v_{p}\right)-\left(v_{p}-v_{-}\right)}{\left(v_{+}-v_{p}\right)+\left(v_{p}-v_{-}\right)}=\frac{v_{+}+v_{-}-2 v_{p}}{v_{+}-v_{-}} .
$$

With this convention we have $-1 \leqslant s \leqslant 1$, with $s=0$ corresponding to a symmetric line. We will employ this definition throughout the present paper. 
Asymmetric line profiles may be represented by a simple modification of eq (2.7), viz,

$$
g(v)= \begin{cases}\left\{1+[v /(1-s)]^{2}\right\}^{-1} & v \leqslant 0 \\ \left\{1+[v /(1+s)]^{2}\right\}^{-1} & v>0\end{cases}
$$

Figure 2 shows typical examples of one symmetric and one asymmetric line fitted by eqs (2.7) and (2.9) respectively.

While eq (2.9) furnishes a reasonably good model function, it has at its peak a discontinuity in the second derivative, which seems physically unrealistic. Hence, in some calculations which are sensitive to behavior at this point, it is preferable to employ the alternative model function

$$
g(v)=(1+\zeta v) /\left(1+v^{2}\right),
$$

which has a qualitatively similar behavior for $\zeta \ll 1$, provided we set $\zeta=2 s$ and translate the origin by $\zeta / 2$.

\subsection{Gaussian Profile}

While a Gaussian profile is not representative of most lines encountered in x-ray spectroscopy, it does furnish another example which is straightforward to compute mathematically. Hence it is of some interest to consider this case in order to see how far the results for a quasi-Lorentzian can be qualitatively applied to other profiles. The Gaussian case is represented by

$$
g(v)=\exp \left[-(\ln 2) v^{2}\right]
$$

in place of eq (2.7). We may then define a quasiGaussian profile analogously to the quasi-Lorentzian one.

\subsection{Variance of Data Points}

To express the variances of the individual data points in terms of the new normalized variables, we note that the intensity $I$ is defined as $I=N / \Delta t$, where $N$ counts have been recorded in an interval $\Delta t$. The corresponding variance of the intensity thus becomes $\sigma_{N}^{2} /(\Delta t)^{2}$ or, by eq $(2.1)$, simply $I / \Delta t$. Employing eq (2.5) to compute the variance of $g$, we find

$$
\sigma_{g}^{2}=\sigma^{2}(I) / I_{p}^{2}=g / I_{p} \Delta t .
$$

In the case of background the calculation is similar except that, in accordance with eq (2.3), we must replace $I$ by $I_{\mathrm{obs}}$, which is given by eq (2.2). For constant background $\mathrm{B}$, the result may be expressed as

where

$$
\sigma_{g}^{2}=(g+\eta) / I_{p} \Delta t
$$

$$
\eta=B / I_{p} .
$$

\section{Centroid}

\subsection{Truncation Procedure}

To complete the definition of the centroid given by eq (1.1) we must specify the truncation limits. Taylor,
Mack, and Parrish [14] have discussed at length several alternative definitions from the viewpoint of crystallographers. They recommended using a specified
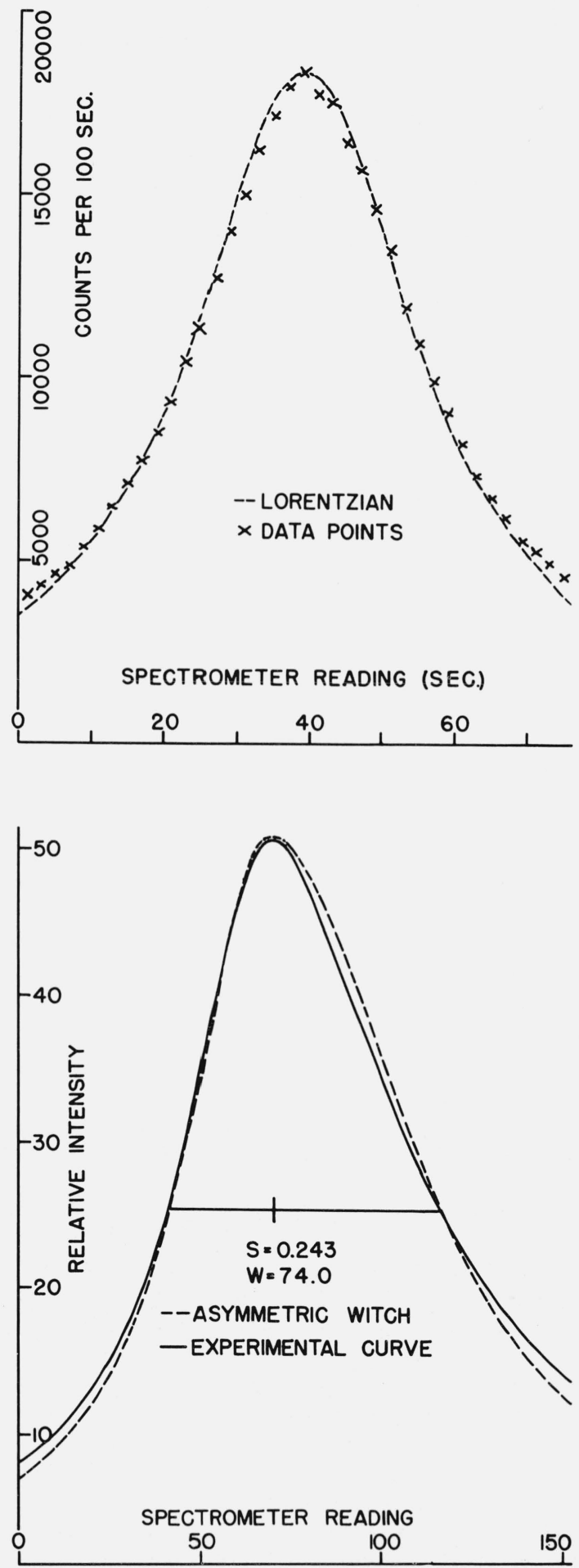

FIGURE 2. (a) Lorentzian curve fitted to experimental data for W $\mathrm{K} \alpha_{1}$ line obtained with a double-crystal spectrometer in $(l,+3)$ position. (b) Model function of eq (2.9) compared with experimental curve for the highly asymmetric $\mathrm{Fe} \mathrm{K} \alpha_{1}$ line. 
interval symmetric about the centroid; this interval may be located by successive approximations. This definition permits a rather straightforward error analysis and will be employed here.

Even if we knew the correct truncation limits exactly, there would be an error in the centroid due to statistical fluctuations in intensities; we will define this as the idealized statistical error, $\epsilon_{c}$, and denote the corresponding standard deviation by $\sigma_{c}$. Since the limits are in fact determined by successive approximations which are in turn influenced by these statistical errors, an additional uncertainty occurs. As demonstrated below, this introduces a multiplicative factor, which will be denoted as the truncation mislocation factor, $f_{c}$. The product of these two quantities then gives the total statistical error, $\left(\sigma_{c}\right)_{\text {tot }}$.

\subsection{Idealized Statistical Error}

For $N$ equally spaced abscissa intervals the centroid definition in eq (1.1) may be rewritten in terms of summations and normalized variables, given by eqs $(2.5-6)$, as

$$
v_{c}=\Sigma_{i} v_{i} g_{i} / \Sigma_{i} g_{i} .
$$

$(1 \leqslant i \leqslant N$ in all summations, unless otherwise specified.) It follows that

$$
\frac{\partial v_{c}}{\partial g_{j}}=\frac{v_{j}}{\sum_{i} g_{i}}-\frac{\sum_{i} v_{i} g_{i}}{\left(\sum_{i} g_{i}\right)^{2}}=\frac{v_{j}-v_{c}}{\sum_{i} g_{i}} .
$$

Since the $g_{j}$ 's are statistically independent with variances given by eq (2.13), the variance of the centroid is now given by

$\sigma_{c}^{2}=\Sigma_{j}\left(\partial v_{c} / \partial g_{j}\right)^{2} \sigma_{j}^{2}=\left(\Sigma_{i} g_{i}\right)^{-2} \Sigma_{j}\left(v_{j}-v_{c}\right)^{2}\left(g_{j}+\eta\right) / I_{p} \Delta t_{j}$.

We may immediately write this in integral form for the case of equal time intervals, noting the $\Delta v=2 \mathrm{~V} / \mathrm{N}$ for the normalized abscissa range $-V \leqslant v \leqslant V$ and that the total counting time $T=N \Delta t$. The result is

$$
\begin{aligned}
\left(\sigma_{c}\right)_{\mathrm{eq}} \Delta t=\left(2 V / I_{p} T\right)^{1 / 2} & {\left[\int_{-V}^{V} g(v) d v\right]^{-1} } \\
& \left\{\int_{-V}^{V}\left(v-v_{c}\right)^{2}[g(v)+\eta] d v\right\}^{1 / 2} .
\end{aligned}
$$

In general, it may be necessary to employ fractional intervals for $\Delta v$ at the end points in order to obtain a range symmetric about $v_{c}$. However, this refinement should not affect the error estimate appreciably, provided $N$ is reasonably large. Furthermore we may frequently bypass the use of fractional intervals by the method given below in section 3.4.

\subsection{Optimization of Statistical Error}

The idealized statistical error may be reduced by proper choice of the time intervals $\Delta t_{j}$. Let us fix the total time $T$, which is given by

$$
\Sigma_{j} \Delta t_{j}=T .
$$

We may then use the method of Lagrangian multipliers to minimize the $j$ summation in eq (3.3) subject to the constraint of eq (3.5) with the result

$$
-\left[\left(v_{j}-v_{c}\right)^{2}\left(g_{j}+\eta\right) /\left(\Delta t_{j}\right)^{2}\right]+\lambda^{2}=0 .
$$

Solving for $\Delta t_{j}$ and employing eq (3.5) to evaluate $\lambda$ yields

$$
\Delta t_{j}=T\left|v_{j}-v_{c}\right|\left(g_{j}+\eta\right)^{1 / 2} / \Sigma_{i}\left|v_{i}-v_{c}\right|\left(g_{i}+\eta\right)^{1 / 2},
$$

where the absolute value sign is used to assure that all time intervals are positive. This equation indicates that, for optimum error, more time should be spent at points on the sides than those at the center; a large background tends to equalize the optimized intervals, as might be expected.

Inserting these values in eq (3.3) and expressing the result in integral form, as done with eq (3.4), we find for the optimized error

$\left(\sigma_{c}\right)_{\mathrm{opt}}=\int_{-V}^{V}\left|v-v_{c}\right|[g(v)+\eta]^{1 / 2} d v /\left(I_{p} T\right)^{1 / 2} \int_{-V}^{V} g(v) d v$.

This result is a special case of that given by Wilson, Thomsen and Yap [12]. From eqs (3.4) and (3.8) we may form the optimization ratio

$$
\frac{\left(\sigma_{c}\right)_{\text {opt }}}{\left(\sigma_{c}\right)_{\text {eq }} \Delta t}=\frac{\int_{-V}^{V}\left|v-v_{c}\right|[g(v)+\eta]^{1 / 2} d v}{\left\{2 V \int_{-V}^{V}\left(v-v_{c}\right)^{2}[g(v)+\eta] d v\right\}^{1 / 2}} .
$$

The above expression is simply the ratio of the mean value of the function $\left|v-v_{c}\right|[g(v)+\eta]^{1 / 2}$ to its rootmean-square value. Hence its qualitative behavior can readily be visualized by plotting this function for any specific case.

\subsection{Truncation Mislocation Factor}

Let us define our coordinate system with origin at the true centroid. If the desired truncation limits were known exactly, there would be only the idealized statistical error $\epsilon_{c}$ as discussed in section 3.1. Actually the limits are never exactly known; we may begin with a trial value of $v_{c}^{(0)}$ for the centroid and use this to compute a first approximation $v_{c}^{(1)}$. Similarly successive approximations may be computed, the $k$ th approximation being denoted by $v_{c}^{(k)}$.

In eq (3.1), the denominator is proportional to the area under the profile in the range $-V \leqslant v \leqslant V$; this is insensitive to small location errors for a curve of moderate asymmetry. The numerator represents a first moment and is sensitive to such errors. For the $(k+1)$ th approximation the range is

$$
-\boldsymbol{V}+v_{c}^{(k)} \leqslant v \leqslant V+v_{c}^{(k)} ;
$$


if $v_{c}^{(k)}$ should vanish, we would obtain the idealized statistical error, $\epsilon_{c}$, for the particular curve. Hence eq (3.1) may be rewritten as

$v_{c}^{(k+1)} \approx \int_{-V+v_{c}^{(k)}}^{V+v_{c}^{(k)}} v g(v) d v / \int_{-V}^{V} g(v) d v \approx \epsilon_{c}+r_{c} v_{c}^{(k)}$,

where

$$
r_{c} \equiv V[g(V)+g(-V)] / \int_{-V}^{V} g(v) d v .
$$

Now $r_{c}$ is simply the ratio of the average height of of the profile at the end points to the average height over the entire range; hence $r_{c}<1$. The recursion relation, eq (3.10), and the initial condition are satisfied by

$$
v_{c}^{(k)}=\epsilon_{c} \sum_{m=0}^{k-1} r_{c}^{m}+r_{c}^{k} v_{c}^{(0)},
$$

which converges to the value $v_{c}$ given by

$$
v_{c}=\epsilon_{c} /\left(1-r_{c}\right) .
$$

The denominator on the right is independent of the particular curve. Hence the standard deviations given by eqs (3.4) and (3.8) (which represent averages over several curves) must be multiplied by a truncation mislocation factor $f_{c}$, i.e.,

$$
f_{c} \equiv\left(\sigma_{c}\right)_{\mathrm{tot}} / \sigma_{c}=1 /\left(1-r_{c}\right) .
$$

The above results also suggest a means of bypassing the successive iterations. For $k=1$, eq (3.12) becomes simply $v_{c}^{(1)}=\epsilon_{c}+r_{c} v_{c}^{(0)}$. Solving for $\epsilon_{c}$ and substituting in eq (3.13) yields

$$
v_{c}-v_{c}^{(0)}=\left(v_{c}^{(1)}-v_{c}^{(0)}\right) /\left(1-r_{c}\right) .
$$

Thus, if the trial value $v_{c}^{(0)}$ is a reasonably good guess, the value of $r_{c}$ may be approximated and $v_{c}$ then computed from the above expression without further iterations.

\subsection{Application to Quasi-Lorentzian Profile}

If the line profile is quasi-Lorentzian (see sec. 2.2), we may substitute eq (2.7) in the various expressions for the centroid to obtain more explicit results. Since $v_{c}=0$ for this model, eq (3.4), for equal time intervals, yields

$$
\begin{aligned}
\left(\sigma_{c}\right)_{\text {eq } \Delta t}=\left(V / I_{p} T\right)^{1 / 2}\left(\tan ^{-1} V\right)^{-1}[ & V-\tan ^{-1} V \\
& \left.+\left(\eta V^{3} / 3\right)\right]^{1 / 2} .
\end{aligned}
$$

This result is essentially the same as that obtained by Pike and Wilson [16]. In figure 3 it has been transformed to the wavelength variable $x$ by means of eq (2.6) and is plotted for various values of range and background.

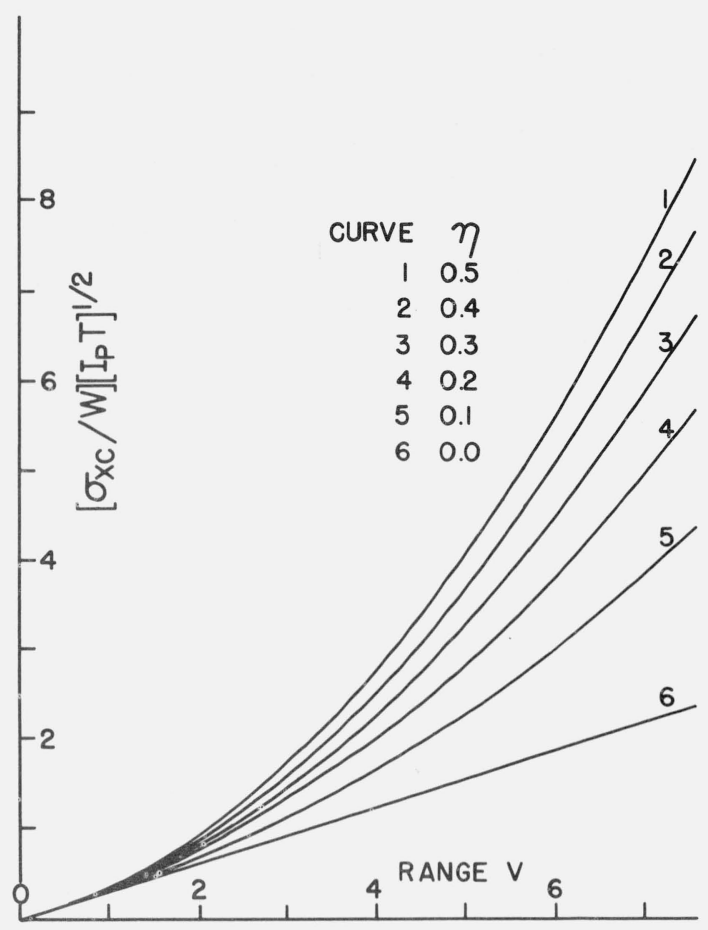

Figure 3. Idealized statistical error of centroid of a quasi-Lorentzian profile as function of truncation range for various values of background parameter $\eta$.

Similarly we may integrate eq (3.9) explicitly for the quasi-Lorentzian case; however, the resulting expression is rather complicated and not particularly informative. For the special case of zero background, the result in simply

$$
\begin{aligned}
& \left(\sigma_{c}\right)_{\mathrm{opt}} /\left(\sigma_{c}\right)_{\mathrm{eq}} \Delta t \\
& \quad=\left[\left(1+V^{2}\right)^{1 / 2}-1\right]\left[V\left(V-\tan ^{-1} V\right)\right]^{-1 / 2} .
\end{aligned}
$$

This ratio varies from 0.86 at $V=0$ to 1.00 as $V \rightarrow \infty$. (Detailed calculations indicate that it is insensitive to background.) Hence only a limited improvement can be achieved by optimizing the data-taking procedure.

The truncation mislocation factor is found by combining eqs (2.7), (3.11), and (3.14), with the result

$$
f_{c}=\frac{\left(1+V^{2}\right) \tan ^{-1} V}{\left(1+V^{2}\right) \tan ^{-1} V-V} .
$$

This factor is unity for infinite $V$, attains a value of 2.75 for $V=1.0$, and approaches infinity as $V \rightarrow 0$.

Idealized statistical errors for the case of zero background and equal time intervals have been calculated from eq (3.16), multiplied by the above factor to obtain the total error, and plotted in figure 4 (after conversion to the wavelength variable $x$ ). This curve reaches a minimum for $V \approx 1$, in which case the total statistical error is given by

$$
\left(\sigma_{x c} / \mathrm{W}\right)_{\mathrm{tot}}=\sigma_{c} / 2=0.81 /\left(I_{p} T\right)^{1 / 2,} \quad(V=1) .
$$


(Note that $V=1$ implies the centroid of a relatively narrow truncated portion of the profile, rather than the centroid definition as used by some crystallographers.) Optimization can reduce this error by only 11 percent, as shown by eq (3.17) and by figure 4 .

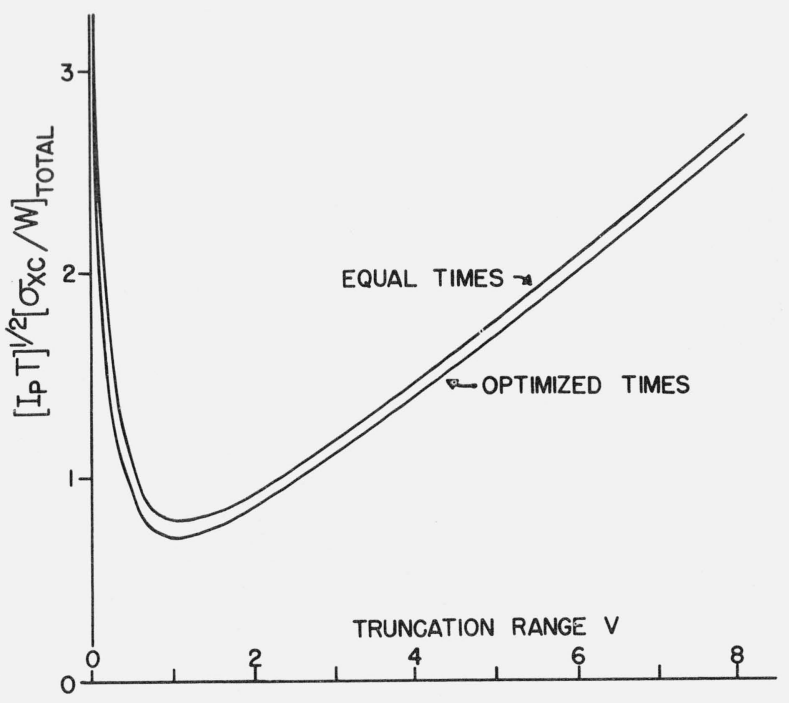

FIGURE 4. Total statistical error of centroid of a quasi-Lorentzian profile as a function of truncation range for equal and for optimized counting time intervals (zero background).

\subsection{Application to Quasi-Gaussian Profile}

We may carry out similar calculations for the quasiGaussian profile defined by eq (2.11). The minimum error occurs at $V \approx 1.8$, for which the numerical factor in eq (3.19) is reduced to 0.62 . In contrast to the Lorentzian case, this range includes over 90 percent of the area under the profile; this is due to the rapid decay of the tails of a Gaussian.

Optimization of time intervals can reduce the above figure by about 8 percent. For large truncation ranges and zero background, optimization could be of crucial importance; the numerical factor would then asymptotically approach 0.68 in sharp contrast with the case of equal intervals, where the error approaches infinity. However, any appreciable background will cause an increase in the error at large ranges and greatly reduce the advantage of optimization.

\subsection{Syst:zmatic Errors}

There are at least two sources of systematic error whose effects may depend upon the choice of wavelength criterion adopted; these are undetected background and the presence of unresolved satellites. Since we consider only the case where truncation limits are taken symmetrically about the centroid, the resulting centroid position must be unaffected by a constant background, even if no correction is made for it. However, if there is an undetected linearly varying background, i.e., if the observed intensity (after the experimental background correction) is given by

$$
g_{\mathrm{obs}}(v)=g(v)+\eta+\mu v,
$$

the linear term will cause a systematic error. Assuming that the curve is almost symmetrical, we may as a first approximation calculate the centroid for a symmetric range about the origin and then apply eq (3.15) to find the centroid of the observed curve. The result may be expressed as

$$
v_{c}+\delta v_{c}=\frac{v_{c}^{(1)}+\delta v_{c}^{(1)}}{1-r_{c}}=\frac{\int_{-V}^{V} v g(v) d v+\left(2 \mu V^{3} / 3\right)}{\int_{-V}^{V} g(v) d v-V[g(V)+g(-V)]},
$$

where $\delta v_{c}$ represents the systematic error due to the background term in $\mu$. For a quasi-Lorentzian profile this term becomes

$$
\delta v_{c}=\frac{\mu V^{3}\left(1+V^{2}\right)}{3\left[\left(1+V^{2}\right) \tan ^{-1} V-V\right]} .
$$

This error is roughly equal to $\mu$ for $V=1$ and to $0.2 \mu V^{3}$ for $V \gg 1$. Thus for $V=1$ and $I_{p} T \sim 10^{6}$, we must have $\mu \sim 10^{-3}$ to keep this systematic error of the same order as the statistical one; for $V \gg 1$, the requirement is clearly far more stringent. It should be reemphasized that $\mu$ refers only to the undetected portion of the background, i.e., it is an error term in the background correction.

A satellite, whose amplitude is likely to be voltage dependent, may be strong enough to cause an appreciable systematic error in the centroid and yet not be resolved. For large truncation ranges, the worst situation occurs with the satellite just short of the truncation limit; its moment arm is then almost equal to $V$. If $W_{s}$ denotes the satellite width and $\alpha_{s}$ the ratio of its amplitude to that of the main peak, the systematic error is roughly $V \alpha_{s} W_{s} / W$. For $V \sim 1$, the worst situation occurs when the satellite peak is appreciably inside the truncation limit; the error is then of the order of $0.5 \alpha_{s}$ and is less sensitive to $W_{s}$.

\subsection{Effect of Asymmetry}

The form of eqs (3.4) and (3.9) indicates that the centroid statistical errors should be insensitive to moderate asymmetries since they depend on the shape only through integral expressions involving the normalized profile. Furthermore it seems clear that the error should not depend on the sign of $s$ (the index of asymmetry defined by eq (2.8)); hence we might expect that the relative change in the standard deviation is of the order of $s^{2}$. This expectation was verified [13] by taking the model function of eq (2.9) with asymmetry $s=0.24$ (corresponding to the $\mathrm{Fe} \mathrm{K} \alpha_{1}$ line) and inserting it in eq (3.4). The resulting error was about 1 percent greater than that for the symmetric quasi-Lorentzian. 
The actual displacement of the centroid from the peak will depend both on the range and on the model function employed. For $V=1$ the displacement $v_{c}-v_{p}$ is approximately $0.67 \mathrm{~s}$ with eq $(2.9)$ and $0.50 \mathrm{~s}$ with eq (2.10). In terms of wavelength these expressions become $0.34 s W$ and $0.25 s W$, respectively.

\section{Median}

\subsection{Computation and Truncation Procedure}

As in the case of the centroid, we must employ a truncated portion of the line profile for a practical determination of the median. For simplicity, we will adopt the same convention, viz, a specified range which is symmetric about the median and which may be determined by successive approximations. The total statistical error will again be given by an idealized statistical error, $\epsilon_{m}$, with corresponding standard deviation $\sigma_{m}$, multiplied by a truncation mislocation factor, $f_{m}$.

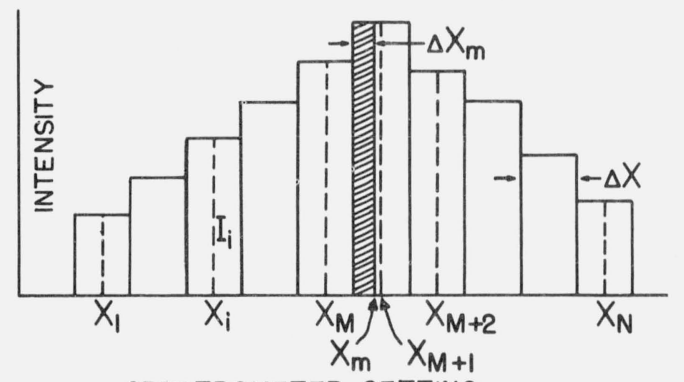

SPECTROMETER SETTING

FigURE 5. Illustration of median located in the $(\mathrm{M}+1)$ th interval, as used in derivation of eq (4.3).

In order to calculate the median error it is helpful to derive a more explicit expression for the median than that given by eq (1.2). Let us pass from the continuous to the discrete case and represent the data by the histogram of figure 5, where each abscissa value corresponds to the midpoint of the base of the corresponding rectangle. If the median is located in the $(M+1)$ th interval, its position $x_{m}$ is defined by

$$
{ }_{m}=x_{M}+(\Delta x / 2)+\Delta x_{m} .
$$

Equation (1.2) now becomes

$$
\sum_{i=1}^{M} I_{i} \Delta x+I_{M+1} \Delta x_{m}=\sum_{i=M+1}^{N} I_{i} \Delta x-I_{M+1} \Delta x_{m} .
$$

Rearranging and inserting the normalized variables defined by eqs (2.5-6) yields

$$
\Delta v_{m}=\left(\Delta v / 2 g_{M+1}\right)\left[\sum_{i=M+1}^{N} g_{i}-\sum_{i=1}^{M} g_{i}\right] .
$$

\subsection{Idealized Statistical Error}

The statistical error in $v_{m}$ is just that in $\Delta v_{m}$. The calculation is similar to that for the centroid in section 3.2 , except that two cases must be considered for the required partial derivatives. For $i \neq M+1$, eq (4.3) yields

$$
\left[\partial\left(\Delta v_{m}\right) / \partial g_{i}\right]^{2}=\left(\Delta v / 2 g_{M+1}\right)^{2} .
$$

For the special case in which $i=M+1$, we obtain

$$
\left[\partial\left(\Delta v_{m}\right) / \partial g_{M+1}\right]=-\left(\Delta v / 2 g_{M+1}^{2}\right)\left[\sum_{i=M+2}^{N} g_{i}-\sum_{i=1}^{M} g_{i}\right] .
$$

The summations may now be eliminated through the help of eq (4.3); it follows that

$$
\left[\partial\left(\Delta v_{m}\right) / \partial g_{M+1}\right]=\left(\Delta v-2 \Delta v_{m}\right) / 2 g_{M+1} .
$$

By figure $5,0 \leqslant \Delta v_{m} \leqslant \Delta v$. Hence it is clear that the right side of eq (4.4) represents an upper limit for $i=M+1$; if $N \gg 1$ we may employ this equation for all $i$ without serious error.

The calculation then proceeds as for the centroid, with the result

$$
\left(\sigma_{m}\right)_{\mathrm{eq}} \Delta t=\left(V / 2 g_{m}^{2} I_{p} T\right)^{1 / 2}\left\{\int_{-V}^{V}[g(v)+\eta] d v\right\}^{1 / 2},
$$

where we have set $g_{M+1} \approx g_{m}$, the ordinate at the median.

\subsection{Optimization of Statistical Error}

The optimization procedure may again be carried out by the method of Lagrangian multipliers. The optimum time intervals are

$$
\Delta t_{j}=T\left(g_{j}+\eta\right)^{1 / 2} / \sum_{i}\left(g_{j}+\eta\right)^{1 / 2},
$$

which yield for the optimization ratio

$$
\frac{\left(\sigma_{m}\right)_{\mathrm{opt}}}{\left(\sigma_{m}\right)_{\mathrm{eq}} \Delta t}=\frac{\int_{-V}^{V}[g(v)+\eta]^{1 / 2} d v}{\left\{2 V \int_{-V}^{V}[g(v)+\eta] d v\right\}^{1 / 2}} .
$$

\subsection{Truncation Mislocation Factor}

Calculation of the truncation mislocation factor is also similar to that for the centroid case. Let the origin be located at the true median position and the idealized statistical error be $\epsilon_{m}$. Then, if the range for the $(k+1)$ th approximation is $-V+v_{m}^{(k)} \leqslant v$ $\leqslant V+v_{m}^{(k)}$, eq $(1.2)$ is approximated by

$$
\begin{array}{r}
v_{m}^{(k+1)} \approx\left(1 / 2 g_{m}\right)\left[\int_{0}^{V+v_{m}^{(k)}} g(v) d v-\int_{-V+v_{m}^{(k)}}^{0} g(v) d v\right] \\
\approx \epsilon_{m}+r_{m} v_{m}^{(k)},
\end{array}
$$


where

$$
r_{m} \equiv\left(1 / 2 g_{m}\right)[g(V)+g(-V)]
$$

The factor $r_{m}$ represents the ratio between the average ordinate at the end points and the ordinate at the median position; it will normally be substantially less than unity. Hence the same analysis as for the centroid follows and the truncation mislocation factor has the same form as eq (3.14), i.e., $f_{m}=1 /\left(1-r_{m}\right)$. Equation (3.15) is also applicable to bypass the need for successive approximations, with $v_{m}$ and $r_{m}$ replacing $v_{c}$ and $r_{c}$.

\subsection{Application to Quasi-Lorentzian Profile}

For the case of a quasi-Lorentzian line (which implies that $g_{m} \approx 1$ ), eqs (2.7) and (4.7) give

$$
\left(\sigma_{m}\right)_{\mathrm{eq}} \Delta t=\left\{V\left(\tan ^{-1} V+\eta V\right) / I_{p} T\right\}^{1 / 2} .
$$

This equation is illustrated in figure 6. If this result is reexpressed in terms of the total number of counts for the case of zero background, it agrees with the calculation of Knowles [19] within the approximation of the latter.

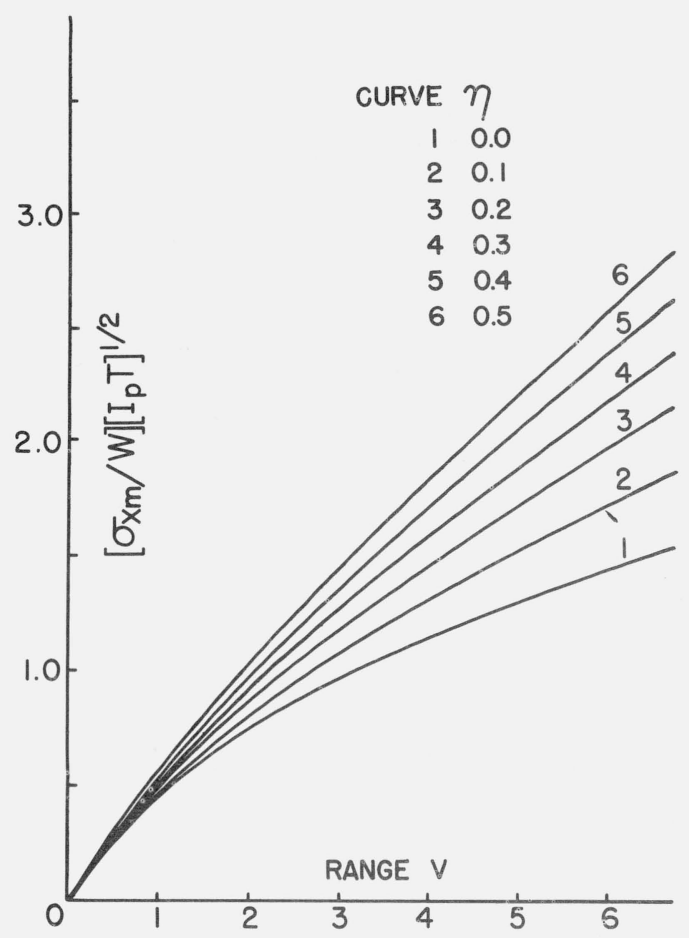

FIGURE 6. Idealized statistical error of median of a quasi-Lorentzian profile as function of truncation range for various values of background parameter $\eta$.

Equation (4.9) may easily be integrated for the case of zero background to yield

$$
\frac{\left(\sigma_{m}\right)_{\mathrm{opt}}}{\left(\sigma_{m}\right)_{\mathrm{eq}} \Delta t}=\frac{\ln \left[V+\left(1+V^{2}\right)^{1 / 2}\right]}{\left[V \tan ^{-1} V\right]^{1 / 2}} .
$$

The factor $r_{m}$ defined by eq (4.11) is simply $\left(1+V^{2}\right)^{-1}$; the truncation mislocation factor then becomes

$$
f_{m}=1 /\left(1-r_{m}\right)=\left(1+V^{2}\right) / V^{2} .
$$

Assuming zero background, we may calculate the total statistical error for the case of equal time intervals by combining eqs (4.12) and (4.14); the optimized case is obtained through multiplication by the ratio given in eq (4.13). The results are plotted in figure 7 . For equal time intervals we find that the minimum error occurs for $V \approx 1.2$ and is

$$
\left(\sigma_{x m} / W\right)_{\mathrm{tot}}=\sigma_{m} / 2=0.88 /\left(I_{p} T\right)^{1 / 2}, \quad(V=1.2) .
$$

Optimized time intervals reduce this figure by only about 1 percent.

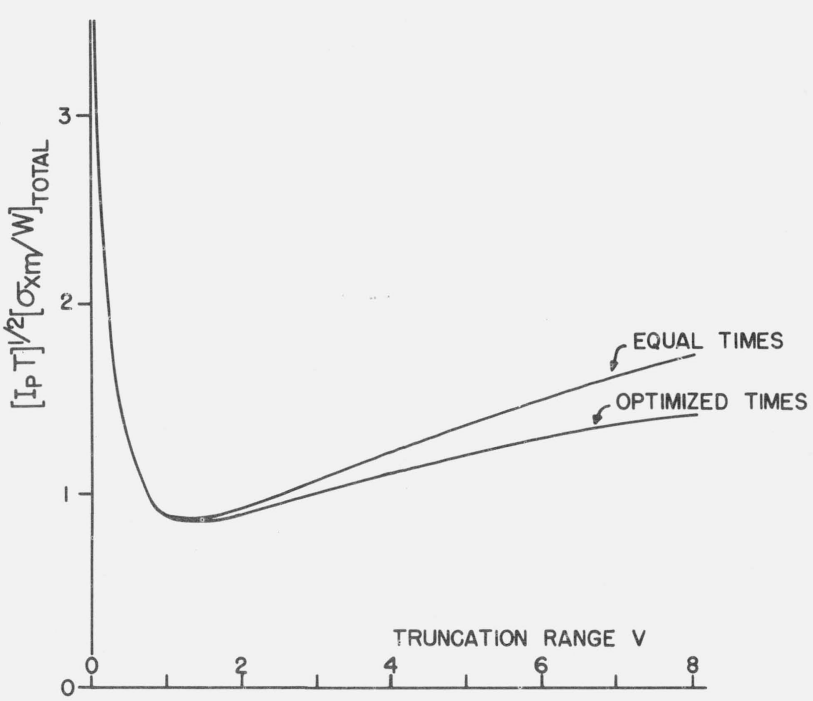

FIGURE 7. Total statistical error of median of a quasi-Lorentzian profile as a function of truncation range for equal and for optimized counting time intervals (zero background).

\subsection{Application to Quasi-Gaussian Profile}

When the results for the median are applied to a quasi-Gaussian profile, the minimum error occurs for $V \approx 1.7$, with the numerical factor in eq $(4.15)$ becoming 0.76 . Optimized time intervals can reduce this figure by about 5 percent. As in the case of the centroid, optimization appears to be of great significance for large truncation ranges and zero background; the numerical factor then approaches asymptotically a value of 0.75 . However, this advantage would be greatly reduced by the presence of any appreciable background.

\subsection{Systematic Errors}

Like the centroid, the median is immune to any systematic error from a constant background, pro- 
$>$ vided that the truncation limits are defined as in sec. 4.1. A linearly varying background $\eta+\mu v$ may be shown to produce a systematic error $\delta v_{m}$ equal to $\mu$ for $V=1$ and approaching $\mu V^{2} / 2$ for $V \gg 1$.

For a large truncation range and a satellite with a width comparable to that of the main line the worst systematic error occurs when the satellite is cenrtered at $\pm V / 2$. In this case substantially all its area lies to one side of the true median and is included in the integration. This area is $\pi \alpha_{s} W_{s} / W$; inspection of eq (4.3) shows that this will cause a systematic error, $\delta v_{m}$, equal to $\pi \alpha_{s} W_{s} / 2 W$. For smaller ranges the error will be magnified by the truncation mislocation factor; on the other hand it will be reduced by the fact that b not all of the satellite area is included within the limits of integration and the included part has significant portions on both sides of the true median. The net result will usually be to reduce the above estimate quite substantially.

\subsection{Effect of Asymmetry}

As in the case of the centroid, we might expect that the statistical error is insensitive to asymmetry and that the relative change in the standard deviation is of the order of $s^{2}$. This prediction has been tested [13] by a calculation with the $\mathrm{Fe} \mathrm{K} \alpha_{1}$ line, for which $s=0.24$. The standard deviation is within roughly 1 percent of the value for a symmetric line.

For the truncation range $V=1$, the displacement of the median from the peak, i.e., $v_{m}-v_{p}$, is $0.57 \mathrm{~s}$ for eq (2.9) and $0.39 s$ for eq (2.10). For infinite range, the former gives a well-defined median with a displacement of $1.57 \mathrm{~s}$; the latter model then becomes physically absurd, since it implies negative values of $g(v)$.

\section{Geometrical Peak}

\subsection{Orthogonal Polynomial Technique}

In order to determine the geometrical peak of a line profile we must adopt some method of drawing a smooth curve through the data points. When the form of the profile is not known a priori, it seems reasonable to fit the data with an empirical polynomial by the method of least squares, preferably through the use of a computer.

Forsythe [30] has discussed the difficulties which arise with higher degree polynomials, due primarily to roundoff errors, when this method is applied directly. Hence in such cases he has recommended least squares adjustments by means of the orthogonal polynomial technique, which alleviates the roundoff problem. We will adopt this approach and employ a weighting function for the $j$ th point which is inversely proportional to its variance, i.e.,

$$
w_{j}=K / \sigma_{j}^{2} \text {. }
$$

With a slight modification of Forsythe's presentation, a series of orthogonal polynomials may then be defined by

$$
\sum_{j} w_{j} \varphi_{m}\left(z_{j}\right) \varphi_{n}\left(z_{j}\right) \Delta z=\delta_{m n}
$$

where $m$ and $n$ denote the degrees of the respective polynomials and $z$ represents a new abscissa variable normalized such that $-1 \leqslant z \leqslant 1$ for the desired range. If the points are closely spaced and of equal weight, then under this definition the $\varphi_{n}$ 's reduce to the Legendre polynomials. These polynomials may be generated by a variety of techniques, e.g., the Schmidt orthogonalization process or a set of recursion relations given by Forsythe [30].

Let us assume that the true profile $\tilde{g}(z)$ is an $M \mathrm{th}$ degree polynomial, which may be expressed in the form

$$
\tilde{g}(z)=\sum_{n=0}^{M} a_{n} \varphi_{n}(z) .
$$

The true values of the coefficients (the $a_{n}$ 's) are, of course, unknown. However, if we denote the (normalized) observed intensity at the $j$ th point by $g_{j}$ and compute the coefficients by the relation

$$
a_{n}=\sum_{j} w_{j} \varphi_{n}\left(z_{j}\right) g_{j} \Delta z
$$

we can show [13] that the resulting polynomial is a least squares fit to the data.

We may now show that the coefficients are statistically independent quantities with equal variances. Since the $\delta g_{j}$ 's, i.e., the errors in $g$ at the various points, are statistically independent, it follows immediately that the average $\left\langle\delta g_{i} \delta g_{j}\right\rangle=\delta_{i j} \sigma_{j}^{2}$. Combining this with eq (5.4) yields

$$
\begin{aligned}
\left\langle\delta a_{m} \delta a_{n}\right\rangle & =(\Delta z)^{2} \sum_{i} \sum_{j} w_{i} w_{j} \phi_{m}\left(z_{i}\right) \phi_{n}\left(z_{j}\right)\left\langle\delta g_{i} \delta g_{j}\right\rangle \\
& =(\Delta z)^{2} \sum_{j} w_{j}^{2} \phi_{m}\left(z_{j}\right) \phi_{n}\left(z_{j}\right) \sigma_{j}^{2} .
\end{aligned}
$$

Eliminating $\sigma_{j}^{2}$ by eq (5.1) and applying eq (5.2) then shows

$$
\left\langle\delta a_{m} \delta a_{n}\right\rangle=K \Delta z \delta_{m n}
$$

which proves the above statement and gives explicitly the variance of $a_{m}$.

\subsection{Statistical Error}

Let us now express the physical problem in terms of the normalized variable $z$, which was defined such that $-1 \leqslant z \leqslant 1$, i.e.,

$$
z=v / V
$$


and set $g(v)=\tilde{g}(z)$. If we assume equal counting times $\Delta t$ and put $K=1 / I_{p} \Delta t$ in eq (5.1), it follows with the help of eq (2.13) that

$$
w_{j}=1 /\left(g_{j}+\eta\right) .
$$

By this choice the orthogonal polynomials defined by eq (5.2) depend only on the shape of the observed profile and are independent of $I_{p}$ and $\Delta t$. (Note that, in principle, the $g_{j}$ 's in the above expression should be the true values rather than the experimentally observed ones. However, it may be shown [13] that the experimental ones may be used with negligible error under normal conditions.) When the above value of $K$ is substituted in eq (5.6), the result is

$$
\left\langle\delta a_{m} \delta a_{n}\right\rangle=\left(\Delta z / I_{p} \Delta t\right) \delta_{m n}=\left(2 / I_{p} T\right) \delta_{m n}
$$

since $\Delta z=2 / N=2 \Delta t / T$.

To calculate the peak shift due to statistical error we first denote the fitted polynomial by $\hat{g}(z)$. This may be expressed as the sum of the true profile $\tilde{g}(z)$ and an error term $\delta \tilde{g}(z)$, caused by the $\delta a_{n}$ 's, i.e.,

$$
\hat{g}(z) \equiv \tilde{g}(z)+\delta \tilde{g}(z)=\tilde{g}(z)+\sum_{n=0}^{M} \delta a_{n} \varphi_{n}(z) .
$$

If the true peak is at $z_{p}$, for which $\tilde{g}^{\prime}\left(z_{p}\right)$ vanishes, the observed peak will be at a point $z_{p}+\delta z_{p}$ such that

$$
\begin{aligned}
& \tilde{g}^{\prime}\left(z_{p}+\delta z_{p}\right)+\delta \tilde{g}^{\prime}\left(z_{p}+\delta z_{p}\right) \approx \tilde{g}^{\prime \prime}\left(z_{p}\right) \delta z_{p} \\
&+\sum_{n=0}^{M} \delta a_{n} \phi_{n}^{\prime}\left(z_{p}\right) \approx 0
\end{aligned}
$$

with the neglect of second-order terms. By eqs (5.7) and (5.11) it follows that

$$
\frac{\partial v_{p}}{\partial a_{n}}=V\left(\frac{\partial z_{p}}{\partial a_{n}}\right) \approx-\frac{V \phi_{n}^{\prime}\left(z_{p}\right)}{\tilde{g}^{\prime \prime}\left(z_{p}\right)}=-\frac{\phi_{n}^{\prime}\left(z_{p}\right)}{V g^{\prime \prime}\left(v_{p}\right)} .
$$

With the help of eqs (5.12) and (5.9) the standard deviation of the peak becomes

$$
\begin{aligned}
\left(\sigma_{p}\right)_{\mathrm{eq}} \Delta t=\left\{\sum_{n=0}^{M}\left(\frac{\partial v_{p}}{\partial a_{n}}\right)^{2}\left\langle\left(\delta a_{n}\right)^{2}\right\rangle\right\}^{1 / 2} & \\
& \approx \frac{1}{V}\left\{\frac{\sum_{n=0}^{M}\left[\phi_{n}^{\prime}\left(z_{p}\right)\right]^{2}}{I_{p} T\left[g^{\prime \prime}\left(v_{p}\right)\right]^{2}}\right\}^{1 / 2} .
\end{aligned}
$$

(In this expression the quantity $g^{\prime \prime}\left(v_{p}\right)$ in the denominator may be adequately approximated by using the second derivative of the fitted curve, rather than that of the true one.) The $\varphi_{n}$ 's have a rather insensitive dependence on range through the weighting function $w_{j}$ in eq (5.2). Hence the error depends on range chiefly through the explicit factor $V$ in the denominator and through the indirect effect on $M$; the larger the range, the higher will in general be the degree of the polynomial necessary to fit the data.

\subsection{Goodness of Fit}

Since in practice the proper value of $M$ is not known a priori, we must determine this by some empirical test on the fitted curve. One such criterion is the $\chi^{2}$ test (see, for example, Bearden and Thomsen [32]). The residuals for the individual points are $g_{j}-\hat{g}\left(z_{j}\right)$, where $g_{j}$ is the experimental value and $\hat{g}\left(z_{j}\right)$ the value of the smoothed fitted curve at the $j$ th point; the variances are given by eq (2.13).

Thus the expression for $\chi^{2}$ becomes

$$
\chi^{2}=I_{p} \Delta t \sum_{j}\left[g_{j}-\hat{g}\left(z_{j}\right)\right]^{2} /\left(g_{j}+\eta\right) .
$$

When averaged over several runs, the average value $\left\langle\chi^{2}\right\rangle$ should approximate the number of degrees of freedom, which is simply $N-(M+1)$, where $N$ is the number of data points and $(M+1)$ the number of coefficients required for an $M$ th degree polynomial.

While the $\chi^{2}$ test is an excellent criterion for a generally reasonable fit, we may often obtain a more sensitive indication of the best value for $M$ by examining values of the $\left\langle a_{n}\right\rangle$ 's, i.e., the polynomial coefficients in eq (5.3) averaged over several curves, say $N_{0}$ runs. The variance of $\left\langle a_{n}\right\rangle$ is found immediately by dividing eq (5.9) by $N_{0}$. When the magnitude of the average value of a particular coefficient is less than its standard deviation, i.e.,

$$
\left|\left\langle a_{m}\right\rangle\right|<\left(2 / N_{0} I_{p} T\right)^{1 / 2},
$$

it evidently contributes more error than useful information. If this inequality holds for all $m>M$, it will confirm the choice of an $M$ th degree polynomial.

For lines of high symmetry it is usually best to use an even degree polynomial, since the last term improves the fit without appreciably contributing to the error given by eq (5.13). It has been found empirically [13] that for a range $V \approx 1$ and $I_{p} T \sim 10^{6}$ a 6 th degree polynomial is usually adequate to represent the data. This holds for most of the strong $K$ and $L$ lines of medium and short wavelength. The highly asymmetric $\mathrm{Fe} \mathrm{K} \alpha_{1}$ line is somewhat of a borderline case between a 6th and 8th degree fit, with the 8th apparently preferable.

\subsection{Application to Quasi-Lorentzian Profile}

If the line profile is quasi-Lorentzian with a range $V=1$ and $I_{p} T \sim 10^{6}$, theoretical calculations show that a 6 th degree polynomial will satisfy the condition given by eq (5.15). The orthogonal polynomials have been evaluated for this case, with zero background, and substituted in eq (5.13) with the result [13]

$$
\sigma_{x p} / W=\sigma_{p} / 2=1.67 /\left(I_{p} T\right)^{1 / 2}, \quad(V=1, M=6) .
$$


for the standard deviation in terms of $x$. It may be shown that the numerical factor is roughly proportional to $M^{3 / 2}$.

We have checked this equation experimentally with six sets of data on the $\mathrm{W} \mathrm{K} \alpha_{1}$ line, comprising a total of 35 curves. The results, as shown in table 1 , indicate rather good agreement.

TABLE 1. Comparison of theoretical (eq (5.16a)) and experimental standard deviations of the geometrical peak of the W K $\alpha_{1}$ line for a 6 th degree polynomial fit with range $\mathrm{V}=1$.

\begin{tabular}{l|c|c|c|c}
\hline \hline $\begin{array}{c}\text { Crystal } \\
\text { settings }\end{array}$ & $\begin{array}{c}\text { No. of } \\
\text { curves }\end{array}$ & $\left\langle\chi^{2} /\right.$ D.F. $\rangle$ & $\sigma_{\text {exp }}$ & $\sigma_{\text {theor }}$ \\
\hline & & 1.1 & $0.0046 \mathrm{~W}$ & $0.0033 \mathrm{~W}$ \\
$(2,-3)$ & 7 & 1.3 & $.0035 \mathrm{~W}$ & $.0036 \mathrm{~W}$ \\
$(2,-3)$ & 5 & 0.9 & $.0042 \mathrm{~W}$ & $.0037 \mathrm{~W}$ \\
$(2,-3)$ & 7 & 1.1 & $.0038 \mathrm{~W}$ & $.0030 \mathrm{~W}$ \\
$(2,3)$ & 4 & 1.0 & $.0032 \mathrm{~W}$ & $.0033 \mathrm{~W}$ \\
$(2,3)$ & 6 & 1.0 & $.0032 \mathrm{~W}$ & $.0033 \mathrm{~W}$ \\
$(2,3)$ & 7 & & \\
\hline
\end{tabular}

Since the geometrical peak is usually determined with a range $V \sim 1$, background will normally be a rather minor factor. We may obtain an estimate of the background effect as follows: The average value of $g(v)$ over the range $V=1$ is easily computed from eq (2.7) and found to be $\pi / 4$. If we assume equal weights and employ this average value of $g$ in eq (5.8), it follows that for zero background $\varphi_{n}(v)=(\pi / 4)^{1 / 2} \pi_{n}(v)$, where the $\pi_{n}$ 's are the orthonormal forms of the Legendre polynomials. If these are used in eq (5.13), the numerical factor in the above expression becomes 1.68, a change of less than 1 percent. Hence it seems reasonable to adopt this same approximation for a nonzero background. Thus for a range $V=1$, the average value of the observed profile is $(\pi / 4)+\eta$ and eq (5.16a) becomes

$$
\sigma_{x p} / W \approx[1+(4 \eta / \pi)]^{1 / 2}\left[1.67 /\left(I_{p} T\right)^{1 / 2}\right], \quad(V=1) .
$$

\subsection{Optimization of Statistical Error}

The time intervals, i.e., the $\Delta t$ 's, enter eq (5.13) only implicitly through the definition of the orthogonal - polynomials in eq (5.2). Because of this indirect relationship it does not seem feasible to optimize the time intervals for minimum statistical error by the same straightforward method used for the centroid and median. Hence a somewhat less satisfactory alternative approach has been adopted for the peak.

After passing from the sum to the integral in eq (5.2), we may arbitrarily assign a weighting function $w(z)$ to obtain one of the standard sets of orthogonal polynomials. (For a concise summary of several well known examples, see Abramowitz and Stegun [31].) Thus, for example, assigning $w(z)=1$ generates the Legendre polynomials.

Equations (5.1) and (2.13) then fix the relative values of the time intervals required to produce the desired weight. Summing these and equating to the total time $T$ gives the constant $K$ in eq (5.1), which may next be substituted in eq (5.6). It is then straightforward to obtain an error expression analogous to eq (5.13).
We have applied this procedure to a quasi-Lorentzian profile with zero background for three cases, viz, Legendre polynomials and Chebyshev polynomials of the first and second kind. Table 2 shows the results for a 6 th degree polynomial fitted over a range $V=1$, with the previously treated case of equal time intervals listed for comparison. In this instance the latter turns out to give the lowest error.

TABLE 2. Comparison of standard deviations obtained for the geometrical peak with a 6th degree polynomial fit and a range $\mathrm{V}=1$.

$z$ represents the abscissa variable normalized such that the range becomes $-1 \leqslant z \leqslant 1$.

\begin{tabular}{c|l|c}
\hline \hline $\begin{array}{c}\text { Weighting } \\
\text { function }\end{array}$ & \multicolumn{1}{|c|}{ Polynomials } & $\left(\sigma_{x p} / W\right)\left(I_{p} T\right)^{1 / 2}$ \\
\hline & & \\
$1+z^{2}$ & (Equal times - see sec. 5.4) & 1.67 \\
1 & Legendre & 1.68 \\
$\left(1-z^{2}\right)^{1 / 2}$ & Chebyshev (first kind) & 1.76 \\
$\left(1-z^{2}\right)^{-1 / 2}$ & Chebyshev (second kind) & 1.70 \\
\hline
\end{tabular}

Various other ranges and degrees of polynomials were analyzed. In most cases varying the weighting function did not affect the error by more than a few percent. Hence it seems that no significant improvement in the peak error can be obtained by optimizing time intervals.

The calculations described above show that, for the case of Legendre polynomials, we may replace eq $(5.16 \mathrm{a})$ by the approximate expression

$$
\sigma_{x p} / W \approx 0.13(M / V)^{3 / 2}\left(\tan ^{-1} V\right)^{1 / 2} /\left(I_{p} T\right)^{1 / 2}, \quad(\eta=0),
$$

where $M$ is the degree of the polynomial. For the range $0.5 \leqslant V \leqslant 1$ and $M=4,6,8$, this approximation is good to within 5 percent; it may also be used for other weighting functions, but will be a somewhat cruder estimate. It is important to note that $M$ itself is implicitly a function of $V$, as discussed in section 5.2.

\subsection{Application to Quasi-Gaussian Profile}

The peak error was also computed for the quasiGaussian profile as given by eq (2.11). For simplicity, we employed Legendre polynomials according to the procedure outlined in the previous section. With $I_{p} T \sim 10^{6}$ a 4 th degree polynomial gave an adequate fit, as judged by the criterion of eq (5.15). For the range $V=1$ the numerical factor in eq (5.16a) was then reduced to 1.40 .

\subsection{Systematic Errors}

Systematic errors in the geometrical peak due to background and unresolved satellites are easily treated. A linear background, as given by eq (3.20), will have a slope $\mu$; the resulting slope of the observed profile near the origin is approximately $g^{\prime \prime}\left(v_{p}\right) \delta v+\mu$, which vanishes when $\delta v_{p}=-\mu / g^{\prime \prime}\left(v_{p}\right)$ or, in the case of a quasi-Lorentzian, $\delta v_{p}=\mu / 2$. 
The maximum slope of a quasi-Lorentzian satellite with amplitude $\alpha_{s}$ and width $W_{s}$ is $3 \sqrt{3} \alpha_{s} W / 8 W_{s}$. This plays the same role as $\mu$ in the above expression, giving a systematic error $\delta v_{p}=3 \sqrt{3} \alpha_{s} W / 16 W_{s}$ for the worst case of an unresolved satellite.

There is an additional systematic error in the geometrical peak due to the degree of the polynomial used to approximate the profile. If the test of eq (5.15) controls the fit, there might conceivably be any number of omitted orthogonal polynomials whose coefficients (i.e., the $a_{n}$ 's) are just under $\left(2 / N_{0} I_{p} T\right)^{1 / 2}$. It seems reasonable to estimate the error on the basis of the first neglected orthogonal polynomial with an appreciable derivative at the origin; for the quasi-Lorentzian case, this means the first neglected odd coefficient. Comparing the systematic error caused by a neglected coefficient $a_{7}=\left(2 / N_{0} I_{p} T\right)^{1 / 2}$ with the statistical error of the mean of $N_{0}$ runs in which a 6 th degree polynomial was used, we find that they are roughly equal. For a $2 d$ or 4 th degree fit, the systematic error can be still more serious.

However, if both the profile and the range of observation are nearly symmetric, the odd coefficients tend to be much smaller than the preceding even ones. Thus, if $a_{6}$ is barely significant statistically, it is likely that $a_{7}$ is substantially smaller. Hence the above estimate of systematic error is probably overly pessimistic, except perhaps in cases of high asymmetry, e.g., $\mathrm{Fe} \mathrm{K} \alpha_{1}$.

\subsection{Effect of Asymmetry}

The error quoted in sec. 5.4 is not particularly sensitive to moderate values of asymmetry. To check this point the $\mathrm{CuK} \alpha_{1}$ line $(s \approx 0.06)$ and the $\mathrm{Fe} \mathrm{K} \alpha_{1}$ line $(s \approx 0.24)$ were chosen as typical examples of low and high asymmetries. The required orthogonal polynomials were then generated by computer from the original experimental data of Bearden and Shaw [1] and substituted in eq (5.13). As a result, the factor of $1.67 \mathrm{in} \mathrm{eq} \mathrm{(5.16)} \mathrm{was} \mathrm{changed} \mathrm{to} 1.52$ and 1.93 in the two respective cases.

\subsection{Parabolic Fit}

If the profile is fitted only in a relatively small region near the peak, a parabolic fit may be sufficient. In this case the weighting factor in eq (5.8) is substantially constant with the value $(1+\eta)^{-1}$. Thus, in analogy to sec. 5.4, it follows from the integral form of eq (5.2) that the orthogonal polynomials are simply $(1+\eta)^{1 / 2} \pi_{n}(z)$, where the $\pi_{i}$ 's are the normalized Legendre polynomials. The only one presently required is $\pi_{1}(z)=(3 / 2)^{1 / 2} z$. If we assume the line is quasiLorentzian and compute $g^{\prime \prime}(0)=-2$ from eq (2.7), eq (5.13) then yields

$$
\sigma_{x p} / W=\sigma_{p} / 2=0.433(1+\eta)^{1 / 2} / V\left(I_{p} T\right)^{1 / 2} .
$$

The calculation suggests the possibility of a significant reduction in error. Hence a rather detailed study [13] has been made on the range of validity of a parabolic fit. This showed that, on the assumption of a
quasi-Lorentzian line with $I_{p} T \approx 10^{6}$, a parabolic fit will be satisfactory up to $V=0.32$; the right-hand side of eq (5.17) then becomes $1.35 /\left(I_{p} T\right)^{1 / 2}$, an appreciable improvement over the 6 th degree polynomial for $V=1$.

Furthermore, if the true curve is symmetric and if the range of observation is almost symmetric about the true peak, a parabola will locate the peak quite accurately even though it fails to satisfy the $\chi^{2}$ test for goodness of fit. In this case the resulting systematic error is found to be proportional to $\epsilon V^{2}$, where $V$ defines the observed range and $\epsilon$ represents the displacement of the origin from the peak (in units of $v$ ). If, for example, we choose $V=0.5$ and keep $\epsilon \leqslant 0.02$, the systematic error in the computed peak will be about 0.1 percent of the line width.

For an asymmetric line there will be a systematic error even if the range is fortuitously centered about the true peak. Based on the model of eq (2.10), this turns out to be $0.6 s V^{2}$ (in units of $v$ ). Thus for $V=0.5$ and $s=0.05$, the systematic error is about 0.4 percent of the line width. Thus an appreciable asymmetry can preclude use of a parabola over an extended range, as might be expected. This result and eq (5.18) as well are in essential agreement with the calculations of Wilson [22].

\subsection{Optimization of Parabolic Fit}

An effort to optimize the time intervals at each data point for a parabolic fit yields the result [13] that virtually all the time should be divided equally between the two end points. While the proof is somewhat lengthy, the resulting error is easily computed. Let the fitted curve be represented by

$$
\hat{g}(v)=1-A\left(v-v_{p}\right)^{2},
$$

where an approximate value of $A$ has been determined in a preliminary scan. If now readings $g_{2}$ and $g_{1}$ are recorded at $\pm V$, with a time interval $T / 2$ at each, it follows that

$$
g_{2}-g_{1}=4 A V v_{p}
$$

It is clear that the primary factor determining the error in $v_{p}$ is the small difference $g_{2}-g_{1}$, whose variance is found with the help of eq (2.13) as $2[g(V)+\eta] / I_{p} \Delta t$. It follows that

$$
\sigma_{p}^{2}=\frac{2}{(4 A V)^{2}}\left[\frac{g(V)+\eta}{I_{p}(T / 2)}\right]
$$

For a quasi-Lorentzian line with $V \ll 1$, we have $g(\boldsymbol{V}) \approx 1$ and $A \approx 1$; hence for this case

$\sigma_{x p} / W=\sigma_{p} / 2 \approx(1+\eta)^{1 / 2} / 4 V\left(I_{p} T\right)^{1 / 2} . \quad(V \ll 1)$.

This is a distinct improvement over eq (5.17), which represents the case of equal counting times at many points. However, as $V$ is increased, the coefficient $A$ in eq (5.18) will be decreased, and the possible improve- 
ment in accuracy correspondingly diminished. Furthermore, this two-point method assumes the line is exactly symmetrical and provides no internal check on this assumption; this fact would seem to limit its practical application.

\subsection{Profile Known a Priori}

We now turn to the case where the exact form of the profile is known or assumed a priori. The problem then reduces to determining the amplitude, width, and peak position, which may be done by a least squares adjustment.

The present analysis will be restricted to a symmetric line profile, e.g., a Lorentzian; in this case a determination of the peak is, of course, also a determination of the centroid and the median.

Let the given functional form be denoted by $f(v)$. We will assume that the approximate parameters for the line are known from a preliminary scan and that the approximate peak location is used to define the origin of the $v$-coordinate. In forming the dimensionless variables $g$ and $v$, let these approximate values $I_{p 0}$ and $W_{0}$ be employed. The corresponding true values, $I_{p}$ and $W$, may be expressed as $I_{p 0}(1+\gamma)$ and $W_{0}(1+\mu)$ respectively. The true line profile will then become

$$
g(v)=(1+\gamma) f\left(\frac{v-v_{p}}{1+\mu}\right),
$$

where $\gamma, \mu$, and $v_{p}$ are all small compared with unity.

The above equation may be linearized by expanding in a Taylor series and neglecting higher order terms with the result

$$
\Delta g=g(v)-f(v) \approx f(v) \gamma-v f^{\prime}(v) \mu-f^{\prime}(v) v_{p} .
$$

The experimental value of $\Delta g_{j}$ at the $j$ th point is simply the difference between $g_{j}$, the observed value, and $f\left(v_{j}\right)$, the quantity obtained by substituting $v_{j}$ in the given functional form. If the number of points $N>3$, there will now be an overdetermined set of linear equations with unknowns $\gamma, \mu$, and $v_{p}$. This set may be solved by the method of least squares (see, for example, Bearden and Thomsen [32]).

The equation thus obtained by differentiation with respect to $v_{p}$ is

$$
-\Sigma_{i}\left[f_{i} f_{i}^{\prime} / \sigma_{i}^{2}\right] \gamma+\Sigma_{i}\left[v_{i}\left(f_{i}^{\prime}\right)^{2} / \sigma_{i}^{2}\right] \mu
$$

$$
+\Sigma_{i}\left[\left(f_{i}^{\prime}\right)^{2} / \sigma_{i}^{2}\right] v_{p}=-\Sigma_{i} f_{i}^{\prime} \Delta g_{i} / \sigma_{i}^{2},
$$

where $f_{i}=f\left(v_{i}\right)$ and $f_{i}^{\prime}=f^{\prime}\left(v_{i}\right)$. For a symmetric function $f(v)$ with a range of observation almost symmetric about the peak, the first two sums are negligible, at least for the purpose of an error estimate. In this case eq (5.24) reduces to

$$
v_{p}=-\left[\Sigma_{i} f_{i}^{\prime} \Delta g_{i} / \sigma_{i}^{2}\right]\left[\Sigma_{i}\left(f_{i}^{\prime}\right)^{2} / \sigma_{i}^{2}\right]^{-1} .
$$

Since the variance of $\Delta g_{i}$ is simply that of $g_{i}$, i.e., $\left\langle\Delta g_{i} \Delta g_{j}\right\rangle=\sigma_{i}^{2} \delta_{i j}$, it follows that

$$
\sigma_{p}^{2}=\frac{\Sigma_{i} \Sigma_{j}\left[f_{i}^{\prime} f_{j}^{\prime} / \sigma_{i}^{2} \sigma_{j}^{2}\right] \sigma_{i}^{2} \delta_{i j}}{\left[\Sigma_{i}\left(f_{i}^{\prime}\right)^{2} / \sigma_{i}^{2}\right]^{2}}=\left[\Sigma_{i}\left(f_{i}^{\prime}\right)^{2} / \sigma_{i}^{2}\right]^{-1}
$$

This is essentially the same result as obtained by Muller, Hoyt, Klein, and DuMond [18] for a known profile (a "composite line profile") with specified amplitude and width; under the symmetry condition assumed here, the same error is obtained with only approximate values for these two parameters.

The variance, $\sigma_{i}^{2}$, is obtained from eq (2.13); with the restriction that $\eta \ll g_{i}$ throughout the range of observation, we may approximate this as

$$
1 / \sigma_{i}^{2} \approx\left(I_{p} \Delta t_{i} / g_{i}\right)\left[1-\left(\eta / g_{i}\right)\right] .
$$

Since $f(v) \approx g(v)$, the distinction may now be dropped for the error calculation. Equations (5.26) and (5.27) then give

$$
\frac{1}{\sigma_{p}^{2}}=I_{p} \Sigma_{i}\left[\frac{\left(g_{i}^{\prime}\right)^{2}}{g_{i}}-\frac{\eta\left(g_{i}^{\prime}\right)^{2}}{g_{i}^{2}}\right] \Delta t_{i} .
$$

For equal time intervals $\Delta t_{i}=T / N=T \Delta v / 2 V$; passing from the sum to the integral thus yields

$$
\frac{1}{\sigma_{p}^{2}}=\frac{I_{p} T}{2 V} \int_{-V}^{V}\left\{\frac{\left[g^{\prime}(v)\right]^{2}}{g(v)}-\eta\left[\frac{g^{\prime}(v)}{g(v)}\right]^{2}\right\} d v .
$$

We may now evaluate this integral for the case of a Lorentzian profile, given by eq (2.7), and obtain finally

$$
\begin{array}{r}
\left(\frac{\sigma_{x p}}{W}\right)_{\mathrm{eq} \Delta t}=\frac{\sigma_{p}}{2}=\left(\frac{V}{2 I_{p} T}\right)^{1 / 2} \\
{\left[\frac{V\left(V^{2}-1\right)}{\left(1+V^{2}\right)^{2}}+\frac{4 \eta V}{1+V^{2}}\right.} \\
\left.+(1-4 \eta) \tan ^{-1} V\right]^{-1 / 2} .
\end{array}
$$

Figure 8 shows the resulting errors, plotted as a function of $V$ for various values of $\eta$. In all cases the error is large for small values of $V$, decreases to a minimum near $V=1$, and rises slowly thereafter. For the range $V=1$, the error is

$\sigma_{x p} / W=0.80(1+0.73 \eta) /\left(I_{p} T\right)^{1 / 2}, \quad(V=1)$,

which is obviously competitive with the optimum values for the centroid and the median.

A similar analysis may be applied to any known symmetric profile. If the profile is qualitatively similar to a Lorentzian, the error should be comparable to the above result. For a Gaussian profile the numerical factor 0.80 is reduced to 0.76 . Moderate asymmetry will complicate computation in the least squares adjustment, since it will then usually be necessary to solve three simultaneous equations in $\gamma, \mu$, and $v_{p}$; however, it should not greatly change the resulting error.

\subsection{Optimization of Lorentzian}

The sum in eq (5.28) is a linear function of the $\Delta t_{i}$ 's; it will obviously be maximized (and the error 


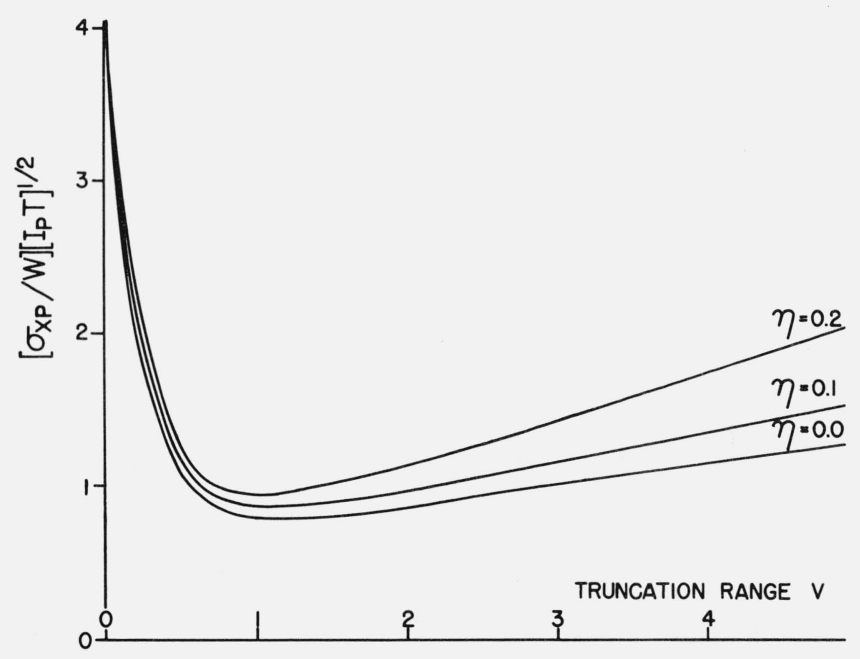

FIGURE 8. Statistical error of peak of a Lorentzian profile (form known a priori) as a function of range for various values of background parameter $\eta$.

Since the profile is symmetric, this also represents the error of the centroid or median.

correspondingly minimized) by dividing substantially all the time between those points for which the coefficient is a maximum. Substitution of the Lorentzian profile function, eq (2.7), and differentiation with respect to $v^{2}$ yields

$$
\frac{d}{d\left(v^{2}\right)}\left[\frac{4 v^{2}}{\left(1+v^{2}\right)^{3}}-\frac{4 \eta v^{2}}{\left(1+v^{2}\right)^{2}}\right]=0 .
$$

With the neglect of second-order terms in $\eta$ the optimized value for $v^{2}$ becomes $(1 / 2)[1-(3 \eta / 4)]$ and it follows that

$$
x_{\mathrm{opt}}=v_{\mathrm{opt}} W / 2= \pm(0.71 W / 2)[1-(3 \eta / 8)],
$$

in agreement with the result of Merrill and DuMond [17].

If time $T / 2$ is spent at each of these two optimized points, the resulting error obtained from eq (5.28) becomes

$$
\sigma_{x p} / W=\left[0.65 /\left(I_{p} T\right)^{1 / 2}\right][1+(3 \eta / 4)]
$$

again with the neglect of second-order terms in $\eta$. The above result represents the lowest statistical error obtained for any case treated in the present paper; however, this method can introduce a serious undetected systematic error if the line profile is slightly asymmetric.

\section{Extrapolated Peak}

\subsection{Midpoint of Single Chord}

The error in the midpoint of a single chord is of interest in itself as well as being a starting point for analysis of the extrapolated peak technique. Suppose a smooth curve $\hat{g}(z)$ has been fitted to the data by the orthogonal polynomial technique described in secs. 5.1 and 5.2. Assume a specified value $G$ and let this be the true value of the curve at $z_{k \cdot 1}$, i.e., $\tilde{g}\left(z_{k+1}\right)=G$; let the fitted curve attain this value at $z_{k 1}+\delta z_{k 11}$, i.e., $\hat{g}\left(z_{k 1}+\delta z_{k 1}\right)=G$. Then it follows from eq (5.10) that

$$
\tilde{g}^{\prime}\left(z_{k 11}\right) \delta z_{k \cdot 1}+\sum_{n=0}^{M} \delta a_{n} \phi_{n}\left(z_{k 1}\right) \approx 0
$$

which yields immediately the value of $\partial z_{k 1} / \partial a_{n}$.

Now let $\bar{z}_{k}$ be the midpoint of a chord drawn at height $G$, intersecting the fitted curve at $z_{1}+\delta z_{k 1}$ on the left side of the profile and at $z_{2}+\delta z_{k 2}$ on the right. Employing eq (6.1) and setting $\tilde{g}^{\prime} \approx \hat{g}^{\prime}$ now yields

$\frac{\partial \bar{z}_{k}}{\partial a_{n}}=\frac{\partial}{\partial a_{n}}\left(\frac{z_{k 1}+z_{k 2}}{2}\right)=-\frac{1}{2}\left[\frac{\phi_{n}\left(z_{k 11}\right)}{\hat{g}^{\prime}\left(z_{k 1}\right)}+\frac{\phi_{n}\left(z_{k 2}\right)}{\hat{g}^{\prime}\left(z_{k 2}\right)}\right]$.

For symmetric curves the two terms in the bracket cancel for even $n$ (since the even order orthogonal polynomials then contain only even terms) and are equal for odd $n$. Thus for this case the above result becomes

$$
\left(\partial \bar{z}_{k} / \partial a_{n}\right)=\left\{\begin{array}{ll}
0 & (n \text { even }) \\
-\phi_{n}\left(z_{k}\right) / \hat{g}^{\prime}\left(z_{k}\right) & (n \text { odd })
\end{array},\right.
$$

where $z_{k}$ denotes either end point of the chord.

Combining this with eqs (5.7) and (5.9) now gives

$$
\begin{aligned}
\sigma_{k}^{2}=\sum_{n=0}^{M}\left(\partial v_{k} / \partial a_{n}\right)^{2} & \left\langle\left(\delta a_{n}\right)^{2}\right\rangle \\
& =\left(2 / \mathbf{I}_{p} T\right) \sum_{n \text { odd }}^{M} V^{2}\left[\phi_{n}\left(z_{k}\right) / \mathrm{g}^{\prime}\left(z_{k}\right)\right]^{2},
\end{aligned}
$$

or

$$
\sigma_{x k} / W=\sigma_{k} / 2=V /\left(2 I_{p} T\right)^{1 / 2}\left\{\sum_{n \text { odd }}^{M}\left[\phi_{n}\left(z_{k}\right)\right]^{2}\right\}^{1 / 2} /\left|\hat{g}^{\prime}\left(z_{k}\right)\right|
$$

for symmetric line profiles.

This expression was evaluated [13] for a quasiLorentzian line with a 6th degree polynomial and a range $V=1$. It is found to give a minimum for $g\left(z_{k}\right) \approx 0.75$, in reasonable agreement with the result of Bačkovský [24], who used uniform weighting. In this case we have

$$
\sigma_{x k} / W=0.95 /\left(I_{p} T\right)^{1 / 2}, \quad(g=0.75) .
$$

This accuracy is obviously competitive with the centroid or median under optimum conditions. However, unless the true line profile is exactly symmetric, it will define an entirely new feature and will introduce a systematic error if used to approximate the peak.

\subsection{Use of Fitted Curve}

If we draw a number of chords as described in the above section, it is possible to fit a smooth "bisector 
curve" through their midpoints with the use of Legendre polynomials and extrapolate this curve to the top of the profile to obtain the peak. This follows a commonly used graphical technique and is shown in figure 1 . With the aid of eq (6.3) we may compute the resulting statistical errors.

Such calculations [13], which are somewhat lengthy, show only minor differences when compared with the result of sec. 5.2 for the geometric peak. For chords equally spaced along the ordinate the error seems to be slightly higher. With chords drawn at equally spaced $v_{k}$ 's along the abscissa, experimental results indicate a slight reduction in statistical error. However, additional sources of systematic error may be introduced by the choice of range in which the chords are constructed, as well as by the polynomial degree of the bisector curve. Hence, while this extrapolation technique is a valuable tool for graphical work, it seems to offer little advantage for computer calculations.

\subsection{Use of Raw Data}

It is possible to bypass the fitted curve and construct the bisector curve directly from the data points on either side of the line profile. The error analysis [13] of this method is somewhat simpler mathematically than the preceding case and hence permitted a more comprehensive study. With a straight line as the bisector curve, the resulting statistical error is competitive with the geometrical peak. In the case of a parabola, this error is increased by roughly 50 percent. Actually it is very doubtful that even a parabola is of sufficiently high degree to represent the bisector curve of a line profile with moderate asymmetry. Thus, except as a possible alternative technique for almost symmetric lines, this approach appears to offer little advantage.

\section{Conclusions}

In general our results are in good agreement with prior workers for cases which have been treated previously. The only possible exception seems to be the suggestion of Porteus and Parratt [21] that the use of the centroid involves an unduly large error; their conclusion appears based on consideration of an infinite truncation range rather than the truncated portions which must be used in practice.

While our experimental results on the peak, as reported in sec. 5.4, are in good agreement with theory, further experiments to verify other predictions would be desirable. In this connection it might be helpful to compare the reproducibility of the difference between two wavelengths (e.g., the $\mathrm{K} \alpha_{1,2}$ doublet) as determined by different criteria.

Our analysis is not, of course, restricted to x-ray line profiles, but applies to any similar problem where the errors in the ordinates are primarily due to counting statistics (e.g., nuclear spectroscopy and Mössbauer studies). Furthermore, even when a different error law holds, the basic method of optimizing counting times, as developed in sec. 3.3, may be of value in suggesting a more efficient data-taking technique. In fact, the possible gain may be greater in other fields than in the $x$-ray problems which we have analyzed.

All of our results may be expressed in the form

$$
\sigma_{x} / W=F /\left(I_{p} T\right)^{1 / 2},
$$

where $F$ is a dimensionless factor of the order of unity. Values for a few representative cases are listed in table 3. (While standard deviations are tabulated, error distributions are normal; hence probable errors can be immediately obtained by multiplying by 0.67 .)

TABLE 3. Statistical errors of wavelength features determined by various methods (zero background).

$\sigma$ represents standard deviation, $W$ the full width of the profile at half intensity, $I_{p}$ the peak intensity, and $T$ the total counting time. The range is specified by the dimensionless parameter $V=2 X / W$, where $-X \leqslant x \leqslant X$.

\begin{tabular}{|c|c|c|c|}
\hline Case & Truncation & $F=(\sigma /)$ & $\left(I_{p} T\right)^{1 / 2}$ \\
\hline 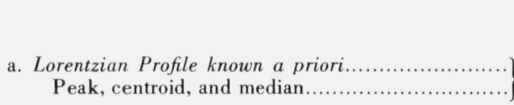 & $\begin{array}{r}0.71 \\
{ }^{*} 1.00\end{array}$ & $\begin{array}{c}\text { Equal } \\
\text { times } \\
0.81\end{array}$ & $\begin{array}{c}\text { Opt. } \\
\text { times } \\
0.65\end{array}$ \\
\hline 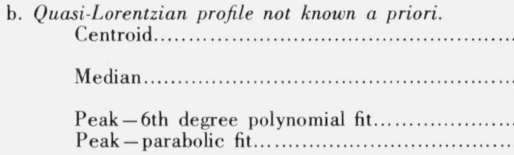 & $\begin{array}{r}* 1.0 \\
6.0 \\
* 1.2 \\
6.0 \\
1.0 \\
0.32\end{array}$ & $\begin{array}{l}0.81 \\
2.11 \\
0.88 \\
1.49 \\
1.67 \\
1.35\end{array}$ & $\begin{aligned} & 0.73 \\
& 2.05 \\
& 0.88 \\
& 1.28 \\
\approx & 1.67 \\
& 0.82\end{aligned}$ \\
\hline
\end{tabular}

*Optimized range for equal counting times.

Obviously it will require the order of $10^{6}$ counts to define a wavelength to within 0.1 percent of the line width. It is also clear that optimization of time intervals can produce only a rather limited improvement in accuracy.

If the line profile is known a priori, it is usually advantageous to make use of this knowledge in calculating the wavelength; e.g., a Lorentzian can be fitted by this method with $F \approx 0.8$. For the Lorentzian or any other symmetric profile known a priori, all three wavelength criteria coincide and any method of locating one locates all. Hence their errors must, of course, be identical. For any known profile of moderate asymmetry the errors should be identical if the width is specified a priori and roughly equal when the width is an adjustable parameter.

In the general case, where the profile form is not known a priori, we may fit a smooth curve to the data by the orthogonal polynomial technique and compute the peak from the fitted curve. (The extrapolation or chord method is a useful graphical technique, but offers no particular advantage in numerical computation, where it can indeed introduce additional systematic error.) With the total counts of the order of $10^{6}$, a 6th degree polynomial is often satisfactory for the range between the half intensity points (i.e., $V=1$ ); resulting values of $F$ range from about 1.6 to 2.1.

We may obtain somewhat lower errors, with a minimum of computational effort, by fitting a parabola over a limited range near the peak. However, extreme caution is necessary when even small asymmetries 
are involved. If the number of counts is substantially lower $\left(\sim 10^{4}\right)$, or if the curve is more nearly Gaussian in shape, a 4th degree polynomial may be satisfactory over the range $V=1$, which will give $F \approx 0.9$.

In many cases, adoption of the centroid or median would promise a reduction in statistical error; these criteria can, in fact, be determined to almost the same precision for a quasi-Lorentzian profile as for a true Lorentzian, known a priori. The reduced error is obtained only for a truncated portion with a range $V \sim 1$. Of course, adoption of either criterion would result in new wavelengths, which would frequently differ significantly from the peak values now in general use.

Definition of wavelength in terms of an arbitrarily defined truncation interval appears lacking in fundamental physical significance. Hence it may seem preferable to attempt to approximate the "true centroid" of the entire profile $(V=\infty)$, as done by some crystallographers, or the true median. In this connection, the median has one obvious advantage, viz, it is well defined mathematically. By contrast the centroid of a quasi-Lorentzian curve involves a logarithmically divergent integral. If the tails are sufficiently symmetric, it may be defined by using symmetric truncation limits in the limiting process; other wise the true centroid simply does not exist mathematically. In any case, when large truncation limits are used, the statistical error may equal or exceed that for the peak of a 6 th degree polynomial.

A linear variation in background will cause a systematic error, about twice as great for the centroid and median as for the peak. (Constant background merely produces an increase in statistical error, which is roughly comparable for all three criteria.) An unresolved satellite may also cause a systematic error. With the range $V \sim 1$, the maximum errors from this source for the centroid or median are again about twice that of the peak. On the other hand, the peak is uniquely subject to an additional systematic error due to the degree of the polynomial used to approximate the observed profile; in some cases this may be of the same order as the statistical error.

We wish to reemphasize that the present study has ignored any errors in correcting for instrumental effects, as stated in Assumption $C$ of section 2.1. In $\gamma$-ray spectroscopy and in x-ray crystallography these corrections may be much greater than the natural line widths. In such cases the statistical error calculations will still give the errors for the respective features of the observed experimental profiles, but these may be completely overshadowed by systematic errors in instrumental corrections. Under these circumstances, statistical counting errors are not particularly relevant in the choice of a wavelength criterion.

Even where instrumental corrections are small, as in the case of $x$-ray spectroscopy, the present error analysis is somewhat inconclusive in indicating a clear choice among the three wavelength criteria considered. Simplicity and historical precedent clearly favor retention of the peak. It seems doubtful that the limited statistical error reduction attainable in some cases by abandoning the peak, seldom greater than a factor of two, is sufficient incentive to warrant a new wavelength definition which would alter many presently tabulated values.

Before reaching a final decision, it would be highly desirable to know how closely each criterion is associated with the term values for the various energy levels. This problem might be attacked by either a theoretical or an experimental approach.

In our present state of knowledge, we recommend that the use of the peak to define $x$-ray wavelength be continued. We further recommend that the geometric peak method be used in preference to extrapolation techniques.

The authors express their thanks to J. A. Bearden for his continuing interest in this work and for numerous helpful discussions. They are grateful to R. T. Cox and Richard D. Deslattes for reading the manuscript and offering suggestions. They are much indebted to William C. Sauder, who began the study of this problem, for his cooperation and for his unpublished calculations. J. W. Knowles, J. O. Porteus, and Lyman G. Parratt were also most helpful in sharing their unpublished work. The National Bureau of Standards has provided much of the necessary support for the project. One of the authors (F. Y. Y.) also gratefully acknowledges support from the Chinese-American Fund of the Johns Hopkins University. Finally thanks are due to Mrs. Mary B. Rowe for typing the manuscript and to Frank Nemec for preparing the figures.

\section{References}

[1] J. A. Bearden and C. H. Shaw, Phys. Rev. 48, 18 (1935).

[2] A. E. Sandström, Handbuch der Physik, 30, Ed. by S. Flügge (Springer-Verlag, Berlin, 1957).

[3] J. A. Bearden, Rev. Mod. Phys. 39, 78 (1967).

[4] J. A. Bearden, A. Henins, J. G. Marzolf, W. C. Sauder, and J. S. Thomsen, Phys. Rev. 135, A899 (1964).

[5] O. Beckman, P. Bergvall, and B. Axelsson, Arkiv Fysik 14, 419 (1958)

[6] J. G. Marzolf, Rev. Sci. Instr. 35, 1212 (1964).

[7] G. Brogren, Arkiv Fysik 8, 391 (1954).

[8] U. Bonse and M. Hart, Appl. Phys. Letters 7, 238 (1965).

[9] J. O. Porteus, J. Appl. Phys. 33, 700 (1962).

[10] W. C. Sauder, J. Appl. Phys. 37, 1495 (1966).

[11] J. S. Thomsen, Bull. Am. Phys. Soc. 10, 1219 (1965).

[12] A. J. C. Wilson, J. S. Thomsen, and F. Y. Yap, Appl. Phys. Letters 7, 163 (1965)

[13] F. Y. Yap, Dissertation (The Johns Hopkins University, Baltimore, Maryland, 1967).

[14] J. Taylor, M. Mack, and W. Parrish, Acta Cryst. 17, 1229 (1964).

[15] J. W. Knowles, $\alpha, \beta$, and $\gamma$ Ray Spectroscopy, I, 203, Ed. by Siegbahn (North Holland Publishing Co., 1965).

[16] E. R. Pike and A. J. C. Wilson, Brit. J. Appl. Phys. 10, 57 (1959).

[17] J. J. Merrill and J. W. M. DuMond, Ann. Phys. (N.Y.) 14, 166 (1961)

[18] D. E. Muller, H. C. Hoyt, D. J. Klein, and J. W. M. DuMond, Phys. Rev. 88, 775 (1952).

[19] J. W. Knowles (private communication).

[20] E. R. Cohen and J. W. M. DuMond, Rev. Mod. Phys. 37, 537 (1965), 
[21] J. O. Porteus and L. G. Parratt (private communication).

[22] A. J. C. Wilson, Brit. J. Appl. Phys. 16, 665 (1965).

[23] K. Beu and D. L. Scott, US AEC Report GAT-T-1374 (1960) (unpublished).

[24] J. Bačkovský, Czech J. Phys. B15, 753 (1965).

[25] J. A. Bearden, Phys. Rev. 43, 92 (1933).

[26] J. J. Merrill and J. W. M. DuMond, Phys. Rev. 110, 79 (1958).

[27] G. Donnay and J. D. H. Donnay, Am. J. Sci. Bowen Vol. (Pt. 1), 115 (1952).
[28] V. Weisskopf and E. Wigner, Z. Physik 63, 54 (1930).

[29] A. Hoyt, Phys. Rev. 40, 477 (1932).

[30] G. E. Forsythe, J. Soc. Indust. Appl. Math. 5, 74 (1957).

[31] M. Abramowitz and I. Stegun, Handbook of Mathematical Functions, ch. 22 (Dover Publications, Inc., New York, 1965).

[32] J. A. Bearden and J. S. Thomsen, Nuovo Cimento 5, Suppl., 267 (1957).

(Paper 72A2-494) 